\title{
Dynamics of flexible fibers in viscous flows and fluids
}

\author{
O. du Roure, ${ }^{1}$, A. Lindner, ${ }^{1,2}$, E.N. Nazockdast ${ }^{3}$, and M.J. Shelley ${ }^{4,5}$ \\ ${ }^{1}$ Laboratoire de Physique et Mecanique des Milieux Heterog'enes (PMMH), \\ ESPCI Paris, PSL University, CNRS, Sorbonne University, \\ and Paris Diderot University, 75005 Paris, France; email: olivia.duroure@espci.fr \\ 2 Global Station for Soft Matter, Global Institution for Collaborative \\ Research and Education, Hokkaido University, Sapporo 060-0808, Japan \\ 3 Applied Physical Sciences, University of North Carolina at Chapel Hill, North Carolina 27514, USA \\ 4 The Courant Institute of Mathematical Sciences, \\ New York University, New York, NY 10012, USA and \\ 5 Center for Computational Biology, Flatiron Institute, New York, NY 10010, USA
}

(Dated: May 23, 2019)

\begin{abstract}
The dynamics and deformations of immersed flexible fibers are at the heart of important industrial and biological processes, induce peculiar mechanical and transport properties in the fluids that contain them, and are the basis for novel methods of flow control. Here we focus on the low Reynolds number regime where advances in studying these fiber-fluid systems have been especially rapid. On the experimental side this is due to new methods of fiber synthesis, microfluidic flow control, and of microscope based tracking measurement techniques. Likewise, there have been continuous improvements in the specialized mathematical modeling and numerical methods needed to capture the interactions of slender flexible fibers with flows, boundaries, and each other.
\end{abstract}

\section{INTRODUCTION AND BACKGROUND}

An important class of micro-scale fluid-structure interactions involves the interactions and deformations of flexible fibers with fluid flows. This is evident in the many biological transport processes, such as microorganismal swimming ([71]) or nuclear positioning in eukaryotic cells ([124]), that involve flexible fibers either actuated or passive. Fibers are the microstructure of many complex fluids - biological, industrial, and synthetic - studied both for their scientific and industrial importance and for their peculiar mechanical responses ([11]). Such suspensions are particularly challenging to study because the suspended fibers have many degrees of freedom in deformation, and can exhibit microscopic instabilities. This makes the interaction of fibers with background flows surprisingly complex. Flexible fibers, both anchored and freely suspended, have been studied of late for the purposes in microfluidic flow control, and when actuated can exhibit complex collective and transport dynamics.

This review focuses on the low Reynolds number regime, as progress on fiber-fluid interactions in this regime has been especially rapid. On the experimental side, this is due to improvements in fiber synthesis and characterization, microfluidic flow control and improved microscope-based measurement and particle tracking techniques. By taking advantage of the relative simplicity of the Stokes equations (as opposed to Navier-Stokes), combined with adaptive resolution and fast summation approaches, there has been a likewise rapid improvement in numerical methods for simulating the deformations and interactions of fibers with flows, as well as with each other and other immersed structures. Coarse-grained descriptions of fiber assemblies and suspensions remain in their early stages, but their development is being sped by these new numerical methodologies.

In outline, we first discuss the current state-of-art in experimental synthesis and measurement techniques, and in numerical methods for dynamical simulation fiber-fluid interactions. We then describe the results of both experiment and theory for the dynamics of free and anchored fibers, followed by a review of the current state of research in many-fiber/fluid systems. We close with a discussion of future directions.

\section{Setting the stage}

Here we briefly introduce a few important physical parameters and relations. Consider a slender elastic fiber of length $L$, of circular cross-section with radius $a$ (hence $\epsilon=a / L \ll 1$ ), and flexural rigidity $E=Y I$ with $Y$ the material Youngs modulus and $I$ the areal moment of inertia $\left(I=\pi a^{4} / 4\right)$. This fiber is immersed in a Newtonian fluid of shear viscosity $\mu$ with the fluid motion characterized by a strain-rate $\dot{\gamma}$. Neglecting inertial forces, three important forces are at play: Brownian forces $\sim k_{B} T / L$ ( $k_{B}$ is the Boltzmann constant and $T$ the temperature), drag forces $\sim \mu \dot{\gamma} L^{2}$, and elasticity forces $\sim E / L^{2}$. For most of the work reviewed here, though not all, viscous drag and elasticity forces dominate Brownian forces. That predominance requires that $l_{p} / L \gg 1$ and $P e=8 \pi \mu \dot{\gamma} L^{3} / k_{b} T \gg 1$, where $l_{p}=E / k_{b} T$ is the persistence length of the fiber against thermal fluctuations, and the Péclet number, $P e$, is the ratio of viscous to Brownian forces. Taking water as the solvent, a fluid strain-rate of $\dot{\gamma}=1 \mathrm{~s}^{-1}$ and a fiber of $L=4 \mu \mathrm{m}$ we 
find $P e \sim 400$. For fibers of a length greater than a few microns $P e \gg 1$ and center of mass diffusion can thus always be neglected compared to advection by viscous flow. Now for a material modulus of $Y=1 G P a$ and an aspect ratio $\epsilon=10^{-2}$ we find $l_{p} / L \sim 10^{5}$ and Brownian forces can be neglected over elastic forces. This is the case of most of the synthetic fibers treated in this review. Decreasing the aspect ratio to $\epsilon=10^{-3}$ and $Y$ by an order of magnitude reduces $l_{p} / L$ to $l_{p} / L \sim 1$ and shape fluctuations resulting from Brownian forces will become important. This is the case of semi-flexible polymers as for example actin filaments.

Finally, while this dimensionless parameter will appear naturally later in the review, we introduce $\tilde{\eta}=8 \pi \mu \dot{\gamma} L^{4} / E c$, where $c=-\ln \left(e \epsilon^{2}\right)$, as the ration between viscous and elastic forces, a control parameter in many fiber-fluid problems.

\section{EXPERIMENTAL TECHNIQUES}

A limiting factor in experimentation of fiber-fluid interactions is achieving good control on fibers in terms of their shape, dimensions, and mechanical properties as well as good flow control. Even if low Reynolds number experiments can be carried out at macroscopic scales by using highly viscous suspending fluids, it is much more comfortable and advantageous to work at microscopic scales where microscopy and microfluidics can be combined to perform reliable experiments. The microscopic approach requires microfabrication techniques, smart choice of the microchannel geometry, and ways to track the particle and its deformation while transported. All these aspects will be detailed in the following discussion.

\section{A. Fiber synthesis and properties}

Reliable experiments require fibers that have controlled dimensions and high aspect ratios as well as known and reproducible mechanical properties. Recent fabrication techniques that can be directly implemented into the channels are specially well-suited for this goal.

\section{Fiber fabrication}

The fabrication of microparticles with non-spherical shapes has received much attention in recent years, but elongated objects require specific approaches. Electro-spinning can be an efficient technique to fabricate fibers of typical diameters $\sim 200 \mathrm{~nm}$ and lengths of $1-100 \mu \mathrm{m}$ (Fig. 1i) with controlled mechanical properties ([65, 72, 93]). Microfluidics has opened new routes to tailor elongated objects. Using the control over channel geometry given by soft lithography, it is possible to form a jet of a solution that will solidify either by photo-polymerization or by diffusion of small molecules from the surrounding fluid (Figs. 1a\&b) ([17, 62, 85, 99, 104]). Another option is to photo-polymerize a photo-sensitive solution, directly in the microchannel, through a mask which determines the object shape (Figs. 1ce). The presence of oxygen close to the PDMS walls prevents polymerization in these regions keeping the fabricated object free to flow in the microchannel $([8,55])$. Finally, self-assembly of colloids is a reliable technique that produces very flexible, high aspect-ratio particles but their sedimentation due to their high density can be limiting (Fig. 1h) $([8,47,151])$.

Another attractive strategy is to rely on biology for controlled and monodisperse elongated objects. Actin filaments, semi-flexible polymers resulting from the polymerization of globular protein, are models for flexible Brownian fibers ([53, 69, 76]). Microtubules are another candidate. Bacterial colonies can also form elongated objects that allow fluid-structure interaction problems to be studied $([2,116,117])$.

More complex shapes can be produced. In 2D, approaches based on self-assembly or photo-lithography can be implemented (Figs. 1c-e and [72]). 3D fabrication is more challenging, and attention has focused on microhelices given their similarity to bacterial flagella. Electrospinning is a widespread technology (reviewed in [125]); The spontaneous formation of helices from flat ribbons is an elegant alternative ([106]) (Fig. 1f). Recent developments in 3D printing using two-photon excitation allows sub-micron resolution but is currently limited to rigid objects (Fig. $1 \mathrm{~g}$ ).

\section{Mechanical Characterization}

The mechanical properties of macro-scale fibers can be measured by rheometry or by the bending of the fiber under its own weight (see Fig. 1j). When working with elastomers these two measurements are in good agreement ([112]). Beam-bending measurements can be implemented in a microfluidic channel to perform in situ measurements (see fig.1k) ([32]). 
Fabrication of elongated objects

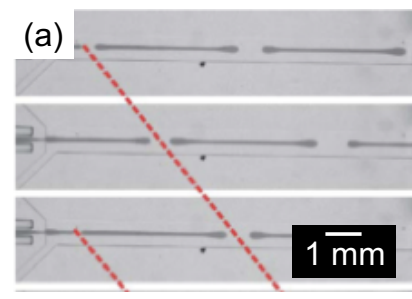

(c)

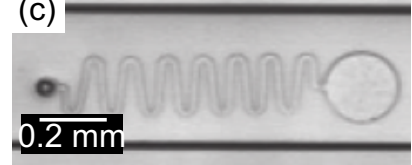

(e)

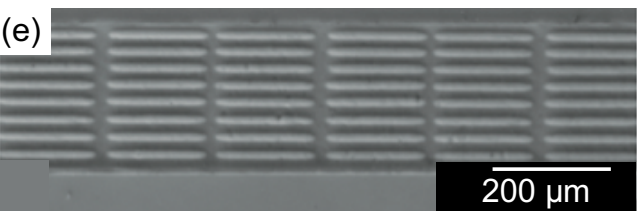

(b)

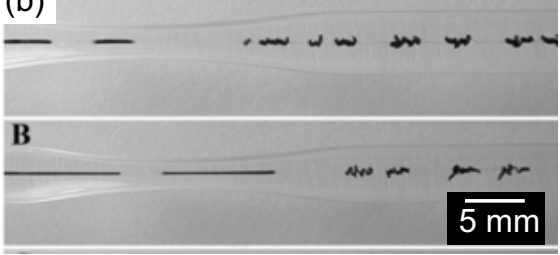

(d)
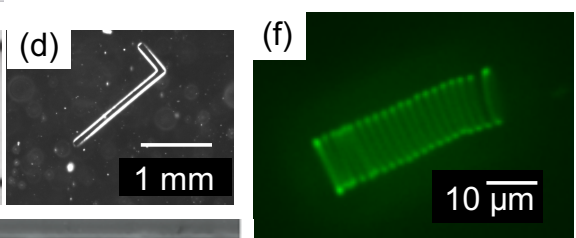

(g)

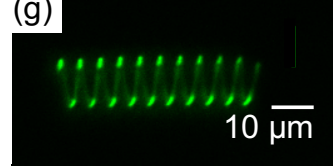

(h)
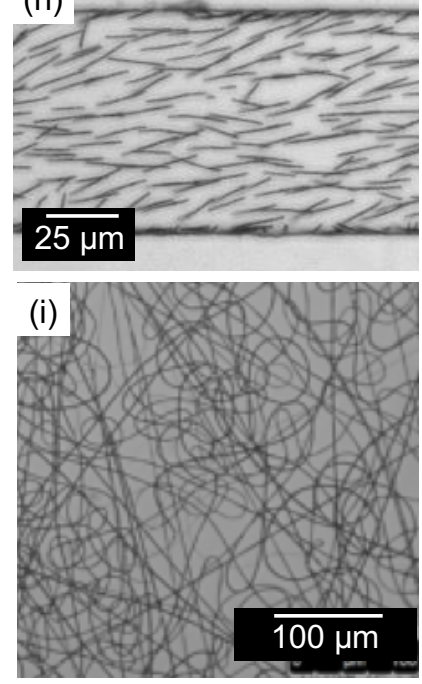

Mechanical properties
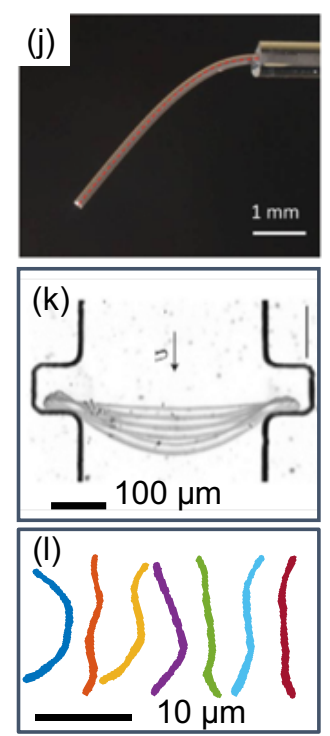

FIG. 1. Fibers, their fabrication, and their mechanical properties. (a) to (e) Objects directly fabricated in microfluidic channels: jets are polymerized either by light (a) [99] or by chemicals (b) [85]. (c) to (e) particles synthesized in a microchannel by photopolymerization of photosensitive solution through a mask. (c) [3], (d) credit: J. Cappello and M. Daïeff, (e) [8] (f) Flexible helix formed spontaneously from asymmetric ribbon, credit: M. Daïeff. (g) Microprinted helix, credit: F. Tesser and J. Laurent. (h) Magnetic fiber suspension fabricated by self-assembly of magnetic colloids, [8]. (i) Electrospun fibers, [93]. (j) to (l) Characterization of mechanical properties. (j) Bending of a macroscopic fiber under its own weight, [111]. (k) Superimposed shapes of a photopolymerized fiber deformed by increasing flow rates, [32]. (1) Bending fluctuations of actin filament, credit: Y. Liu.

When the smallest dimension of the fiber is a micron or less, Brownian fluctuations can be large enough to induce bending deformations. In this case the flexural rigidity $E$ can be expressed as $l_{p} k_{b} T$, with $l_{p}$ the persistence length, and an elegant way to measure $E$ is to extract $l_{p}$ from the analysis of many filament configurations (fig. 1l). This approach is common for biological semi-flexible polymers like actin or microtubules ([44]) and has also been used for tiny electrospun filaments $([93])$.

\section{B. Flow control}

At large spatial scales, working at low Reynolds number requires long-time experiments with low-velocity flows of highly viscous liquids. However, at small scales low Reynolds numbers are easily achievable even with low viscosity fluids. For this regime the channel geometry determines completely the flow and thus different flows can be obtained depending on the device design.

To avoid flow complexity, studies are often done using linear extensional or shear flows (Fig. 2a\&b). Experimentally, working with linear shear flow is only possible in a Couette geometry if the gap is small compared to the cylinder diameters (Fig. 2a). In channel flows, pure shear can be approximated by Poiseuille flow if the object size is much smaller than the channel height (Fig. 2a) ([76]). Cross-slot geometries generate extensional flows but with small spatial extension. An elegant but difficult way of overcoming this limitation is to keep the object at the stagnation point by adjusting the pressures in the different entries as was implemented by [122] and later by [66] (see Fig. 2b). A more robust way is to optimize numerically the exact geometry to obtain a purely extensional flow over a larger distance even in the presence of the walls (Fig.2b) $([54,153])$.

Numerical simulations are sometimes done in 2D due to their relative simplicity, while in experiments $2 \mathrm{D}$ flows are not always easy to achieve. One option is to use a Hele-Shaw geometry, in which lateral dimensions are much larger than the cross-channel dimension (Fig. 2c). On a microscope, the vertical configuration allows a nearly $2 \mathrm{D}$ flow to be generated in the observation plane above the boundary $([54])$.

Flows can be controlled by syringe pumps imposing flow rates, or by pressure control devices. Pressure control is well adapted to low velocity and shear rate. Particle velocimetry techniques are used to quantitatively determine local velocities and thus shear rates. 
(a) Shear flows

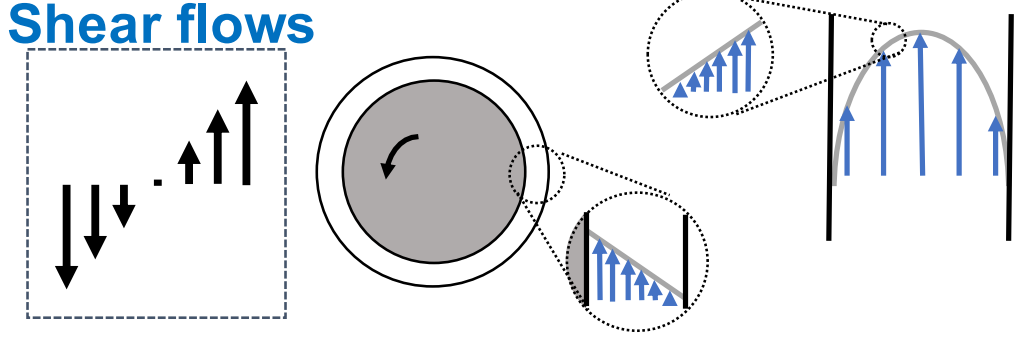

(b) Extensional flows

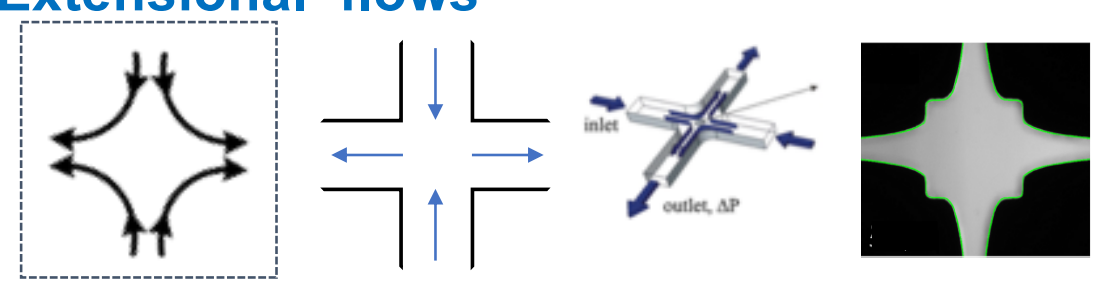

(c) 2D-3D

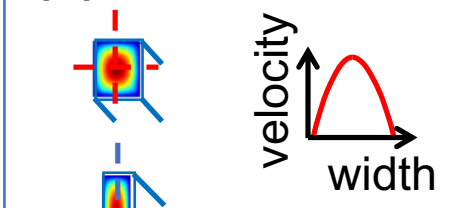

(d) Microfluidic rheometers

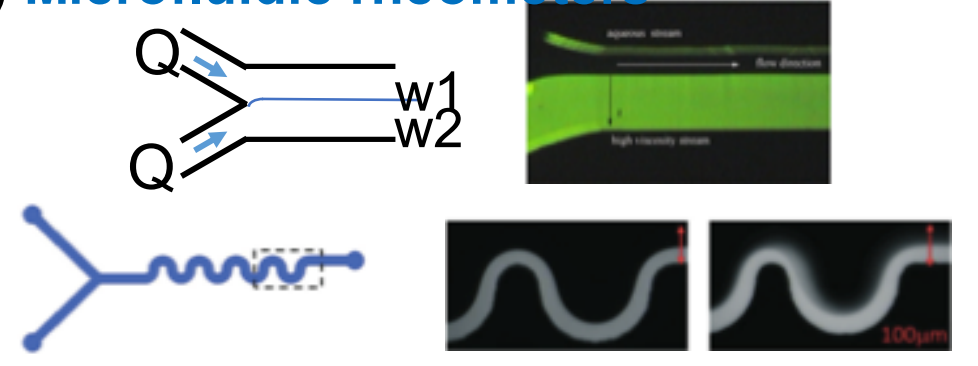

(e) Particle tracking

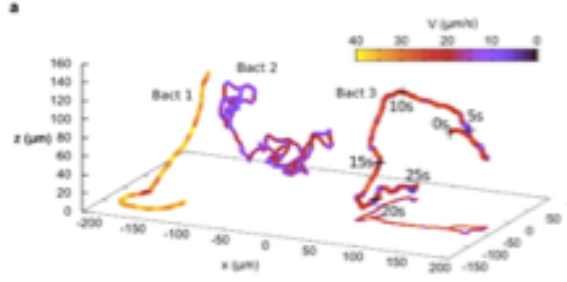

FIG. 2. Flow control and fiber tracking. Experimental realization of (a) shear flow, and (b) extensional flow. (a) From left to right, velocity profile of pure shear, schemes of a Couette flow, and Poiseuille flow in a channel which locally approximate pure shear. (b) Velocity profile of an extensional flow, scheme of a cross-slot channel, experimental realization, [66], optimized cross-slot geometry giving a larger area with constant extension rate, [54]. (c) Flow geometry in 3D. From top to bottom, square channel, vertical and horizontal Hele-Shaw cell. Velocity profiles corresponding to (top, red) a Poiseuille flow and (right, blue) a plug flow. (d) Microfluidic rheometers. Top row, Y-channel is well-suited to measure small variation of viscosity, [42]. Bottom row, serpentine channels can be used to study elastic turbulence in non-Newtonian fluids, [152]. (e) 3D Lagrangian tracking of bacteria in a microchannel, [24].

A new development in the field are microfluidic rheometers that access the rheological properties of complex suspensions of various types ([75]) (Fig. 2d). Their main advantages are their sensitivity to low variations in viscosity and normal stresses and the possibility to directly observe the flowing particles within the device.

\section{Particle observations and tracking}

Typically, fiber deformations and trajectories need to be measured simultaneously even though they occur at different spatial scales: Images with high-magnification are necessary to obtain good resolution of fiber deformations whereas trajectories are inherently large-scale. In a microchannel, bright field, phase contrast, and fluorescence microscopy are usually best suited to acquire sufficient quality images of fiber deformations. The use of high-magnification and a high-numerical-aperture objective is in general required. In addition, a long working distance can be very useful to observe filaments far from the bottom of the chamber where the coupling to the wall is negligible. At macro-scale, the 3D-shape may be reconstructed from images taken by two cameras or by the smart use of mirrors.

To track an object along its trajectory at the macro-scale, automated motion of the camera can be used. Keeping a fiber in the field of view in a microfluidic channel requires that the microscope stage moves at or near the fiber velocity. In combination with a fast camera this avoids image blurring. Following trajectories in 3D becomes necessary when objects do not follow streamlines as in cross-stream migration, or for active particles. In this case, one can use 3D 
tracking algorithms like those developed to follow swimming bacteria $([24,110])$.

\section{MATHEMATICAL MODELING AND SIMULATION METHODS}

The interaction of flexible fibers with Stokesian fluids has a special structure for which specialized mathematical and computational methods have been developed. The most basic is local slender-body theory (SBT), which gives a leading-order local relation between elastic and drag forces and is a mainstay for analysis. Nonlocal interactions arising from fluid incompressibility can be captured through use of higher-order, and more complex, slender-body formulations, or through other approaches such as immersed boundary methods, bead-rod models, or regularized Stokeslet methods. Here we first discuss the nonlocal SBT formulation, as it is compact and amenable to fast solution methodologies.

\section{A. Nonlocal Slender Body Theory}

The appeal of nonlocal SBT is its reduction of filament-fluid and filament-filament interactions to relatively simple dynamics equations for filament centerlines. For this we assume that the fluid is described by the incompressible Newtonian Stokes equation:

$$
\mu \Delta \mathbf{u}-\nabla p=\mathbf{0} \quad \& \quad \nabla \cdot \mathbf{u}=0
$$

with $\mu$ the shear viscosity, $\mathbf{u}$ the fluid velocity, and $p$ the pressure. Generally, solutions to the Stokes equations can be found using boundary integral methods ([108]), where the fluid velocity is represented as a distribution of fundamental solutions to the Stokes equations on all immersed and bounding surfaces. The fundamental solution corresponding to a $\delta$-function of force is given by the Stokeslet tensor: $\mathbf{G}(\mathbf{x})=\frac{1}{8 \pi \mu} \frac{\mathbf{I}+\hat{\mathbf{x}} \hat{\mathbf{x}}}{|\mathbf{x}|}$, where $\hat{\mathbf{x}}=\mathbf{x} /|\mathbf{x}|$.

The flows produced by the motion of slender fibers are treated especially as their surface integrals can be reduced, through asymptotics, to integrals of Stokeslets along their center-lines ([46, 64, 67]). Specifically, consider $N$ fibers, each of radius $a$ and lengths $L_{n}$, with center-line positions $\mathbf{X}^{n}(s, t)$ for $n=1, \ldots, N$, with $s$ the $\operatorname{arclength}\left(0 \leq s \leq L_{n}\right)$. Each fiber is assumed to be slender, i.e., $\epsilon_{n}=a / L_{n} \ll 1$, and to exert a force per unit length $\mathbf{f}^{n}(s, t)$ on the surrounding fluid. Given a background flow $\overline{\mathbf{u}}$ the fluid velocity at a point $\mathbf{x}$ within the fluid is, to leading order, given by distributions of Stokeslets along fiber center-lines:

$$
\mathbf{u}(\mathbf{x})=\overline{\mathbf{u}}(\mathbf{x})+\sum_{m=1}^{N} \mathbf{u}_{F}^{m}(\mathbf{x}) \quad \text { where } \quad \mathbf{u}_{F}^{m}(\mathbf{x})=\int_{0}^{L_{m}} \mathbf{G}\left(\mathbf{x}-\mathbf{X}^{m}\left(s^{\prime}\right)\right) \cdot \mathbf{f}^{m}\left(s^{\prime}\right) d s^{\prime}
$$

[46] derives higher-order corrections in terms of integrals of Stokes doublets.

The self-induced velocity of a fiber is not found by direct evaluation of Eqs. (2) upon that fiber. This yields a logarithmically divergent integral. Instead, fiber self-induction is a matter of careful asymptotic matching $([46,64,67])$. Such analyses give the velocity of the centerline $\mathbf{X}^{n}(s)$ as

$$
\mathbf{V}^{n}(s)=\overline{\mathbf{u}}\left(\mathbf{X}^{n}\right)+\sum_{m=1, m \neq n}^{N} \mathbf{u}_{F}^{m}\left(\mathbf{X}^{n}\right)+c_{m}\left(\mathbf{I}+\mathbf{X}_{s}^{n} \mathbf{X}_{s}^{n}\right) \mathbf{f}^{m}+\mathbf{K}_{n}\left[\mathbf{f}^{n}\right]
$$

where $\mathbf{X}_{s}$ is the tangent vector and subscript $s$ denotes a partial derivative. Here $c_{n}=-\ln \left(\epsilon_{n} e^{2}\right)$ is an asymptotically large parameter, and $\mathbf{K}_{n}$ is an $O\left(\epsilon^{0}\right)$ linear operator that contains a "finite-part" integral of Stokeslets that captures fiber self-interactions. The use of this integral in computation is discussed by [137] who show that it must be appropriately regularized both to maintain asymptotic accuracy and to control the growth of short wavelength modes treated inaccurately by slender-body theory.

Equation (3) relates the fiber velocity to the fiber forces acting upon the fluid. As a dynamics problem, this system is closed by specifying the fiber elastic forces and identifying a Lagrangian or material variable for the fiber. These are done simultaneously by modeling each fiber as an inextensible Euler-Bernoulli beam:

$$
\mathbf{f}^{n}=\mathbf{f}_{\mathrm{E}}^{n}=-E \mathbf{X}_{s s s s}^{n}+\left(T^{n} \mathbf{X}_{s}^{n}\right)_{s}, \quad 0 \leq s \leq L_{n} .
$$

with $-E \mathbf{X}_{s s s s}$ the bending force per unit length, and $T$ the axial tension with $\left(T \mathbf{X}_{s}\right)_{s}$ the tension force per unit length. Inextensibility identifies $s$ as a material variable, and hence $\mathbf{X}_{t}^{n}(s, t)=\mathbf{V}^{n}$. Inextensibility also generates an auxiliary integro-differential equation for $T$ by imposing $\mathbf{V}_{s}^{n} \cdot \mathbf{X}_{s}^{n}=0$ which follows from differentiating the identity $\mathbf{X}_{s}^{n} \cdot \mathbf{X}_{s}^{n}=1([137])$. 


\section{B. The special case of local SBT}

Local SBT has been the mainstay for theoretical analyses of the dynamics of flexible fibers, and sits at the core of many numerical approaches. In particular, retaining from Eq. (3) only the logarithmic leading order in $\epsilon$ we have a local balance of fluid drag forces, and fiber forces exerted on the fluid:

$$
\mathbf{R}(s, t) \cdot[\mathbf{V}(s, t)-\overline{\mathbf{u}}(\mathbf{X}(s, t), t)]=\mathbf{f}(s, t),
$$

where $\mathbf{R}=\eta\left(\mathbf{I}-\mathbf{X}_{s} \mathbf{X}_{s} / 2\right)$ and $\eta=8 \pi \mu / c$. For an elastic fiber modeled by Eq. (4)) we have a dynamical equation, plus a constraint:

$$
\mathbf{X}_{t}=\mathbf{V} \equiv \mathbf{u}(\mathbf{X}, t)+\eta^{-1}\left(\mathbf{I}+\mathbf{X}_{s} \mathbf{X}_{s}\right)\left[-E \mathbf{X}_{s s s s}+\left(T \mathbf{X}_{s}\right)_{s}\right] \quad \& \quad \mathbf{X}_{s} \cdot \mathbf{V}_{s}=0
$$

The inextensibility constraint generates an elliptic equation for $T$ :

$$
2 T_{s s}-\left|\mathbf{X}_{s s}\right|^{2} T=S,
$$

where $S$ depends upon background flow and the bending force. Given appropriate boundary conditions, $T$ has a unique solution, and Eq. (6) can be used to evolve the fiber's shape, position, and orientation.

Basic scaling: Consider a background flow with a characteristic length-scale $W$ and time-scale $\dot{\gamma}^{-1}$, expressed as $\overline{\mathbf{u}}=\dot{\gamma} W \mathbf{U}(\mathbf{x} / W, \dot{\gamma} t)$. By scaling space on $L$, time on $\dot{\gamma}^{-1}$, and tension $T$ on $E / L^{2}$, Eq. (6) can be given in nondimensional form as

$$
\mathbf{V}=\alpha^{-1} \mathbf{U}(\alpha \mathbf{X}, t)-\tilde{\eta}^{-1}\left(\mathbf{I}+\mathbf{X}_{s} \mathbf{X}_{s}\right)\left[\mathbf{X}_{s s s s}-\left(T \mathbf{X}_{s}\right)_{s}\right]
$$

with $\alpha=L / W$, and where $\tilde{\eta}=8 \pi \mu \dot{\gamma} L^{4} / E c$ is the effective strength of flow forcing. Note that for linear background flows $\alpha$ disappears from the equation. If the tension is negative, reflecting compressive fluid stresses, then in Eq. (8) is seen as a competition of fourth-order diffusion and a second-order anti-diffusion.

\section{Boundary conditions}

Finally, boundary conditions are needed on the tensile and bending forces. For freely suspended fibers, these are the "natural" boundary conditions of having zero applied forces and torques, which simplify to $\mathbf{X}_{s s s}=\mathbf{X}_{s s}=\mathbf{0}$, and $T=0$ on the each end of the fiber. These boundary conditions suit the fiber force containing $\mathbf{X}_{\text {ssss }}$ and $T$ satisfying essentially an elliptic boundary value problem. Having filaments attached (clamped or hinged) to surfaces, mobile or immobile, generates other boundary conditions and explores other dynamics, which are detailed in [96].

\section{Brownian semi-flexible fibers}

Thus far we have assumed the fibers to be rigid enough that thermal fluctuations have negligible effect upon their conformations. For this to hold, the length of the fibers needs to be significantly smaller than their persistence length $l_{p}$. If this assumption is not met (as can be for carbon nanotubes, actin filaments, and wormlike polymers), thermal fluctuations can have important consequences on the overall mechanics of the suspension, and has been the subject of many studies. A review of this topic is beyond the scope here and we refer the interested reader to [11]. Here we only give a limited summary of previous work and the standing challenges.

In the presence of thermal fluctuations Eq. (5) is modified to

$$
\mathbf{R} \cdot\left(\mathbf{X}_{t}-\overline{\mathbf{u}}\right)=-E \mathbf{X}_{s s s s}+\left(T \mathbf{X}_{s}\right)_{s}+\mathbf{f}^{B}(s)
$$

where $\mathbf{f}^{B}$ is the thermal fluctuating force with zero mean and finite variance given by fluctuation dissipation theorem:

$$
\left\langle\mathbf{f}^{B}(s, t)\right\rangle=\underline{0} \&\left\langle\mathbf{f}^{B}(s, t) \mathbf{f}^{B}\left(s^{\prime}, t^{\prime}\right)\right\rangle=2 k T \mathbf{R}(s, t) \delta\left(s-s^{\prime}\right) \delta\left(t-t^{\prime}\right)
$$

The brackets denote ensemble averages. [91] used this formulation to study a 2D Brownian semi-flexible fiber in shear flow. [81] also used this formulation in studies of Brownian flexible fibers in two-dimensional arrays of counter-rotating Taylor-Green vortices and extensional flows.

It follows from Eq. (10) that computing the displacements from thermal fluctuations requires computing $\mathbf{R}^{1 / 2}$. For local SBT $\mathbf{R}^{1 / 2}$ can be computed analytically. For non-local SBT, the resistance tensor, $\mathbf{R}$, is nonlocal and depends 
on the position of all fibers. Numerically, this means computing the square root of a square matrix with dimensions $N_{T}^{2}=\left(N \times N_{F}\right)^{2}$, where $N_{F}$ is the number of fibers and $N$ is the number of discretization points per fiber. The direct computational of $\mathbf{R}^{1 / 2}$ requires $\mathcal{O}\left(N_{T}^{3}\right)$ operations, which makes simulation of systems beyond a few fibers very expensive. More efficient techniques have been proposed to reduce the computational cost to $N_{T}^{\alpha}$ with $\alpha$ varying between $1<\alpha<2$, depending on the conditions. In a recent development [37] use Ewald summation to reduce the computational cost to $O\left(N_{T}\right)$.

Several other works have modeled Brownian filaments by using bead-rod/spring models for inextensible polymeric filaments. The main complexity involves imposing the inextensibility constraint. Specifically, it turns out that very stiff springs and inextensible rods differ in their degrees of freedom and hence in the resulting equilibrium statistical mechanics [38]. [57] showed that a psuedo-potential force needs be applied to a connection with a very stiff spring in order to exactly recover the behavior and statistical physics of an inextensible connection.

\section{Fast Numerical Methods for SBT}

[137] developed a stable and numerically tractable version of nonlocal SBT for flexible filaments with free-ends, and devised specialized quadrature schemes for the regularized finite-part integral in $\mathbf{K}[\mathbf{f}]$ of Eq. (3). The fibers' centerlines were discretized uniformly and derivatives evaluated using a second-order accurate finite difference scheme, while trapezoidal rule was used for evaluating the remaining integrals. For time-stepping, a backward differentiation finite difference scheme was used and the bending forces were treated implicitly to remove temporal stiffness, while the tension equation? which imposed inextensibility of the fibers? was treated explicitly.

[96] extended the work of [137] to a platform suitable for simulating a substantially larger number of fiber, to $O(1000)$ or more. They used a Chebyshev basis for the representation of fiber position and to compute high-order derivatives with spectral accuracy. They used implicit-explicit time discretization and treated both bending and tensile forces implicitly. As a result, they observed a three order of magnitude improvement in the maximum stable time-step in their numerical experiments, compared to the explicit treatment of tension by Tornberg \& Shelley. They solved the linearized system of equations using GMRES ([118]) with a Jacobi or block Jacobi preconditioner. A kernel independent fast multipole method (FMM) is utilized for fast computation of nonlocal hydrodynamic interactions via fast matrix? vector products, yielding a computational cost of $O(M)$ operations per time-step, where $M$ is the number of unknowns. The entire computational scheme is parallelized and scalable to many computational cores, which allowed simulation of $O(1000)$ semi-flexible filaments.

\section{E. The Regularized Stokeslet Method}

A popular method for solving the Stokes equations is the method of regularized Stokeslets of [20] which uses superpositions of regularized fundamental solutions. [39] used a superposition of regularized Stokeslets and Rotlets to simulate the dynamics of driven flagellae. In their study, a flagellum is a network of flexible springs, and a helical shape so composed is driven by a torque at its base. In a study also modeling flagella (and cilia) [127] developed a version of regularized Stokeslets that utilizes a boundary-element approach to the discretization and incorporates the presence of walls (see also [63]). [9] use a regularized representation of a one-dimensional curve of two-dimensional Stokeslets to simulate the nonlocal dynamics of flexible, slightly extensible fibers. [100] have combined the regularized Stokeslet method for evolving slender rods with the internal mechanics of an elastica with intrinsic twist and curvature. And, in theoretical work, [21] have developed a asymptotically consistent slender-body theory based on using regularized center-line forces.

\section{F. The Immersed Boundary Method}

The immersed boundary method (IBM) of [105] has also been applied to this class of problems. In this method, a filament is discretized by Lagrangian markers connected by spring elements, and their relative displacements by fluid motions are used to calculate the filament's elastic response. These elastic forces are then distributed onto a background grid covering the fluid volume, and are used as forces acting upon the fluid, thus modifying the fluid flow that displaces the markers [74]. The advantage of the IBM is that detailed immersed mechanical structures can be simulated, but at the cost of having to solve the flow equations in the entire fluid volume. Other volume-based methods have also been used in this context, including finite element/volume methods [88], and Lattice Boltzmann $[145]$. 
Within the immersed boundary framework, for a fiber to have a physical width the volume grid size needs to be several times smaller, making the method computationally expensive for simulating slender bodies such as fibers and thin disks. To overcome this, [97] employed an adaptive-mesh version of the IBM [48] to study the dynamics of flexible fibers as models, in part, for diatom chains (diatoms are unicellular phytoplankton). For this, they constructed fibers that are composite structures made of alternating segments that mimic the structure of diatom chains (see Fig. 4c). While a simple beam model, like Eq. (8), describes its bending deformations well, the results of compressive strain are not. [141] used their parallel implementation of the IBM to simulate a suspension of up to 256 semi-flexible fibers in shear flow. Their analysis of parallel performance shows their algorithm is weakly scalable. Recently, [129] implemented a fourth-order accurate IBM for studying flows over arbitrary smooth domains. A combination of this with adaptive-grid immersed boundary [48] might improve the computational efficiency and accuracy of immersed boundary in simulating slender objects in flows.

\section{G. Bead-and-Rod Models}

While closely related to methods based upon SBT or the immersed boundary method, bead-and-rod models have a somewhat independent lineage. In bead-and-rod models, a flexible fiber is represented as a one-dimensional chain of linked rigid bodies (e.g. spheres, spheroids, rods) that experience local drag and interact with each other through short-range forces (e.g. repulsion, lubrication, friction), while sometimes neglecting long-ranged hydrodynamic interactions or only including a subset of them. A recent review of these methods is found in [52]. In recent work [26] have elaborated upon basic bead models by introducing a new Lagrange multiplier method to impose constraints, and consider several flow problems - Jeffery orbits, buckling in shear, actuated swimming filaments - by using an approximate accounting of Stokesian hydrodynamics.

\section{FREE FIBERS IN FLOW}

How fibers are buckled by flow is central to much of the interesting nonlinear dynamics observed in simulations and experiments of fiber motion. Here we first discuss a prototypical situation - a straight fiber moving in a linear straining background flow - where buckling arises as an instability. We then discuss other prototypical problems such as morphological transitions of fibers transported in linear shear flows and more complex flow geometries where the coupling of fiber deformation and transport can be observed.

\section{A. Fiber morphology in simple flows}

Here we discuss the shape dynamics of fibers in linear shearing and straining flows.

\section{Buckling instabilities in compressive flows}

Because of its simplicity, local SBT is usually the preferred tool for studying linear stability of immersed fibers and is used here to illustrate the buckling instability of free fibers in the simplest of cases.

A perfectly straight isolated fiber will remain straight in any incompressible and linear flow, even as the fiber is translated and rotated. Hence, consider the background flow $\mathbf{U}(\mathbf{x}, t)=\mathbf{A}(t) \cdot \mathbf{x}$ with $\operatorname{tr}(\mathbf{A})=0$. A straight fiber can be represented as $\mathbf{X}(s, t)=\mathbf{X}_{c}(t)+s \mathbf{e}(t)$ with $\mathbf{X}_{c}$ its center point, and e a unit orientation vector. Inserting this form into Eqs. (8) \& (7), and applying the "natural" conditions of zero total force and torque, yields

$$
\dot{\mathbf{X}}_{c}=\mathbf{A} \mathbf{X}_{c}, \quad \dot{\mathbf{e}}=\left(\mathbf{I}-\mathbf{e e}^{T}\right) \mathbf{A e}, \quad \bar{T}=-\frac{\tilde{\eta}}{4}\left(\mathbf{e}^{T} \mathbf{E e}\right)\left(s^{2}-1 / 4\right)
$$

where $\mathbf{E}=\left(\mathbf{A}+\mathbf{A}^{T}\right) / 2$ is the symmetric rate-of-strain tensor. Hence, the rod center is carried with the local flow, the orientation vector obeys Jeffrey's equation ([61]), and the tension $\bar{T}$ is quadratic in $s^{2}$ with its sign determined by the orientation of $\mathbf{e}$ relative to the principle axes of $\mathbf{E}$. Thus, if the fiber is aligned with compressive straining of the flow then the tension is negative and hence compressive. This is the condition required for a buckling instability.

The most straightforward case is given by a simple straining flow $\overline{\mathbf{u}}=(x,-y)$ where the straight fiber is aligned with the $y$-axis (i.e., $\mathbf{e} \equiv \hat{\mathbf{y}}$ ). This choice maximizes flow compression ([150]) and is free of explicit time-dependence from 

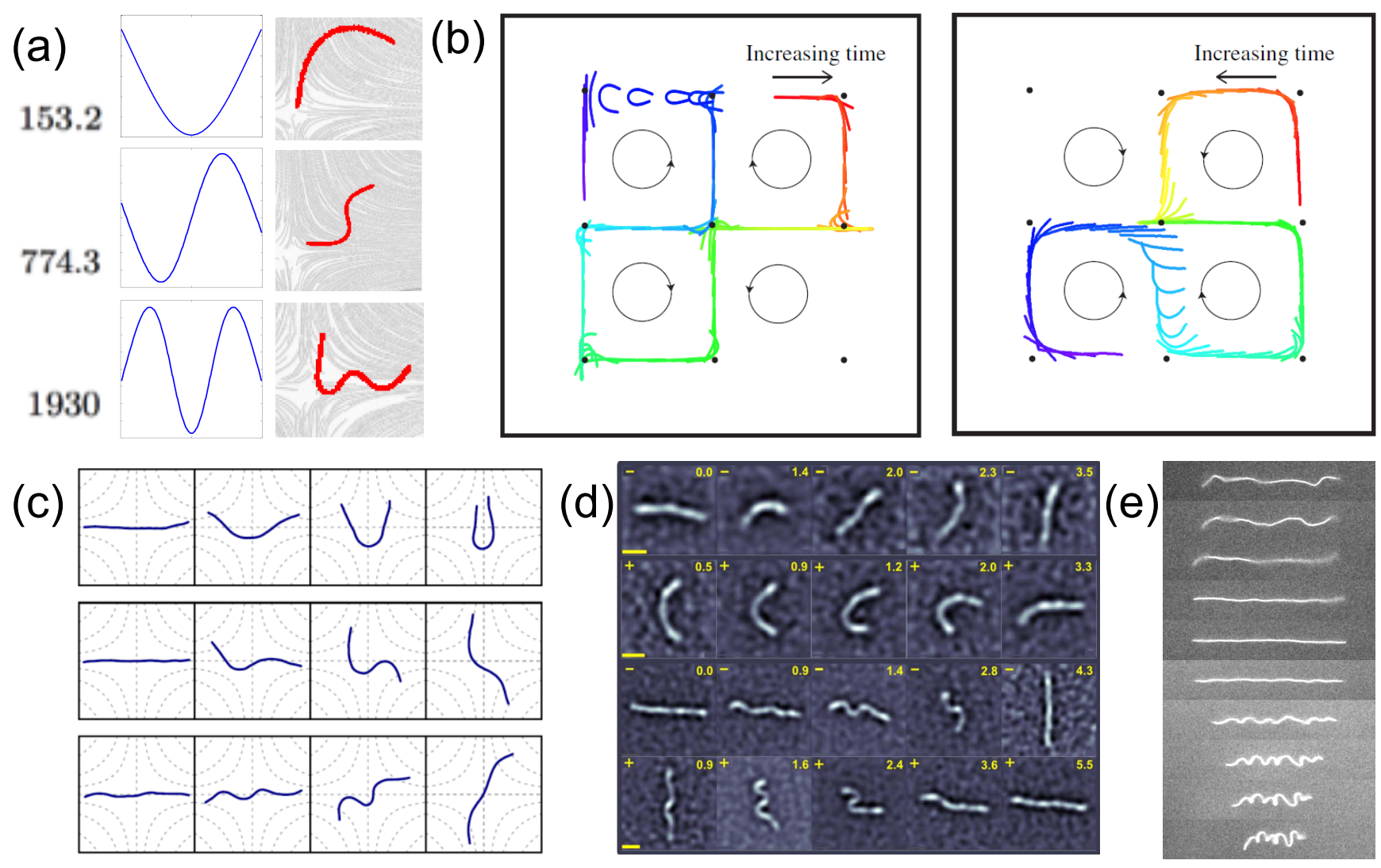

FIG. 3. Fiber buckling. (a) Critical values of the control parameter $\tilde{\eta}$ and fiber shapes of buckling modes from linear stability analysis and experiments, [112]. (b) Flexible fiber evolving in an array of hyperbolic stagnation points, $\tilde{\eta} \approx 2000$, [112]: Left, simulations using SBT, right, experiments with a polymeric centimetric fiber. (c) \& (d) Brownian fiber maintained at a stagnation point. $\tilde{\eta}$ increases from top to bottom. (c) Numerical simulations using SBT, [82]. (d) Experiments using actin filaments in a microfluidic device, [66]. (e) Actin filament transported in a converging/diverging hyperbolic microchannel experiencing elongation followed by compression at $\tilde{\eta}=7.910^{5}$, credit: Y. Liu.

the background flow. This leads to a variable coefficient eigenvalue problem for the growth or decay of an in-plane perturbation $w$ to the straight fiber:

$$
\sigma w=w+s w_{s}+\frac{1}{4}\left(s^{2}-1 / 4\right) w_{s s}-\tilde{\eta}^{-1} w_{s s s s}
$$

where $w_{s s}=w_{s s s}=0$ at $s= \pm 1 / 2$. With an eigenvalue solver we can track the system's eigenvalues and eigenfunctions as $\tilde{\eta}$, the effective viscosity or strain-rate, is increased. For small $\tilde{\eta}$ the straight fiber is stable to perturbations. With increase in $\tilde{\eta}$ we find the successive crossing to the right half-plane of eigenvalues coupled to eigenfunctions associated with increasingly higher order bending modes. The first three crossings occur at $\tilde{\eta}_{1}=153.2, \tilde{\eta}_{2}=774.3$, and $\tilde{\eta}_{3}=1930([112])$, and their associated eigenfunctions are shown in Fig. 3a (left panel). The respective eigen-shapes are the classical U-, S-, and W-shaped buckling modes.

In experiment it is nontrivial to achieve a linear straining flow and reasonably long residence times for a fiber moving in it. One possibility is to use the approach of a fiber to a stagnation point. Fiber buckling has been investigated in a macroscopic realization of such a system by [139] who used centimetric soft elastomer fibers. These fibers move in a viscous quasi-2D cellular flow, an array of counter-rotating vortices surrounding hyperbolic stagnation points. Above a critical value of $\tilde{\eta}$, fibers are observed to buckle (Fig. 3b (right)) at or near stagnation points. With increasing $\tilde{\eta}$ more complex fiber shapes were observed (Fig. 3a (right)), corresponding to the eigen-shapes predicted by the linear stability analysis. Fiber trajectories from experiments and simulations (3b) show fiber transport across the cellular array. Due to the complexity of the flow, buckling does not take place at each passage of a stagnation point, but depends on the exact conformation and position of the approaching fiber ([112]).

Buckling instabilities have also been investigated in the presence of Brownian fluctuations. [82] have shown numerically that Brownian fluctuations play a minor role on the buckling instability and serve mainly to broaden the 
threshold of the transitions towards the different buckling modes. [66] investigated the deformation of a micrometric actin fiber held at a stagnation point created in a microfluidic cross-slot device. Their experiments as well as numerical simulations by [82] show modes qualitatively similar to the deterministic predictions, albeit with shape fluctuations owing to Brownian fluctuations (see Figs. 3c, d).

The optimal flow geometry for the investigation of buckling instabilities is a hyperbolic channel, where constant extension rates can be achieved over long residence times. This was realized recently using actin filaments transported in an optimized microfluidic flow geometry (sec. II B) and preliminary results can be seen on Fig. 3e. The filament is first stretched in the converging part of the channel where Brownian fluctuations are suppressed by the viscous forces. Then the filament enters the diverging part of the channel where it experiences strong compression leading to higher buckling modes. A similar succession of stretching and coiling has been observed in microchannels with constrictions for actin filaments $([132])$ and polymeric microfibers $([99])$.

\section{Fiber morphology in shear flows}

A linear shear flow is the superimposition of a rotational and a straining flow and, as a consequence, fiber dynamics are a combination of tumbling and periodic deformation. A rigid ellipsoid tumbles periodically in a linear shear flow, while resisting stretching or compression as it rotates through the extensive and compressive quadrants. A flexible fiber on the other hand will react to the straining flow, leading to a transition to a buckling instability as $\tilde{\eta}$ is increased $([7,40,76])$. When $\tilde{\eta}$ is increased further, fibers become too flexible to sustain rotation of the fiber as a whole and a transition to "snaking" motion is observed. Here the fiber remains mainly aligned with the flow direction, but performs a tank trading motion ([26, 41, 53, 76, 97]).

Early experiments by [41] investigated the deformation of millimetric elastic fibers in corn syrup under linear shear in a Couette cell. They were the first to address fiber buckling ([40]) and observed what they baptized "springy rotations" ([41]). For longer fibers they identified "snake" turns as shown on Fig. 4a. The transition to fiber buckling was clearly identified only later in a numerical investigation by [7] for a fiber rotating in a linear shear flow. The first transition to buckling takes place at $\tilde{\eta}=306.4$. This is twice the value $\tilde{\eta}_{1}=153.2$ found for straining flow, as the magnitude of the straining part in shear flow is proportional to only half of the magnitude of the shear flow. The two threshold values are thus effectively identical. Using the local SBT formulation, their numerical simulations studied the very nonlinear shape dynamics of the fiber above the buckling transition (see Fig. 4d). Experiments by [53] using actin filaments in microscopic channel flows have investigated in detail the "snake" turn motions and were the first to describe and model this configuration as a tank trading motion where a narrow bend travels along the filament whose two ends remain mainly aligned with the flow. They also addressed the role of rotational diffusion on the period of Jeffery orbits. Numerous investigations have observed the "snaking" dynamics numerically ([26, 97, 131]) (see Fig. 4c). Only recently have all subsequent regimes been observed (Fig. 4e) and classified (Fig. 4f) as a function of $\tilde{\eta}$ and $l_{p} / L$, by experiments using actin filaments and a numerical investigation using non-local SBT in the presence of Brownian fluctuations ([76]). Special care was taken when designing the experimental flow geometry to assure linear shear conditions (sec. II B). The authors also addressed the transition to the "snaking" mode which is initiated by a buckling instability occurring, under sufficiently strong shear, in a filament that has not yet aligned with the compressive axis. Rotation of the deformed fiber will then align one end with the flow direction whereas the other can bend. The theoretically predicted threshold is in good agreement with experimental observations (Fig. 4f). Well above the onset of the "snaking" motion more complex dynamics such as coiling ([97]), knot formation ([70]), or helical motion $([41])$ were also reported.

\section{B. Fiber morphology and transport in more complex flows}

Here we discuss the shape dynamics of fibers in more complex background flows, such as those arising from confinement or imposition of a background flow through external forcing.

\section{Poiseuille flow and interaction with bounding walls}

Channel (or Poiseuille) flows represent the majority of experimental systems and, unlike linear shear flows, exhibit a shear gradient across the channel width. This can affect fiber morphology when fiber lengths are comparable to the channel dimensions. A first consequence is that buckling instabilities (as described above) are not observed and bending modes dominate. Typical fiber morphologies in Poiseuille flows are reminiscent of "snaking" motions ([53]) (Fig. 4b) and can vary depending on the exact positioning within the flow ([16, 115]) (Fig. 5a,b). Secondly, 

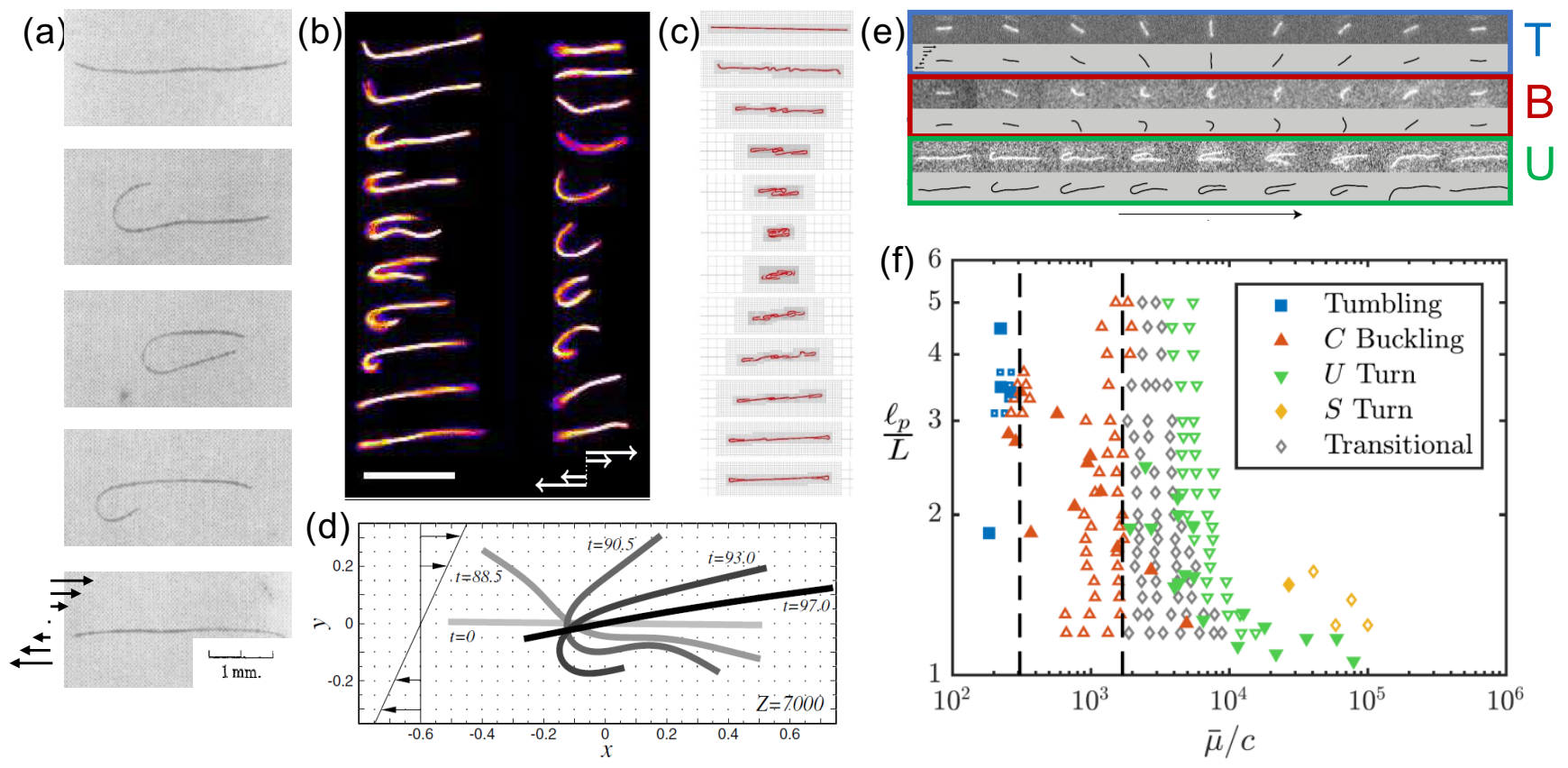

FIG. 4. Fibers in shear flows. (a) Elastomeric fibers performing snake-turns in corn syrup in a simple shear flow, [41]. (b) Actin filaments in a microfluidic geometry, [53]. (c) Complex fiber shapes from simulations using an adaptive version of the immersed boundary method, [97]. (d) Dynamics of a buckling fiber at $\tilde{\eta}=7000$ from simulations using local SBT, [7]. (e) Different morphologies of Brownian fibers with increasing length from experiments using actin filaments (top) and simulations using SBT (bottom), [76]. (f) Phase diagram on filament morphologies. The vertical lines indicate the transitions to buckling and snaking motions, [76].

(a)

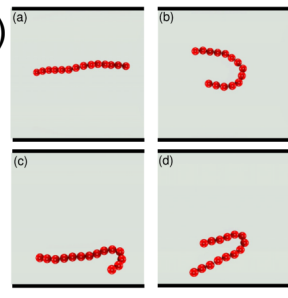

(c)

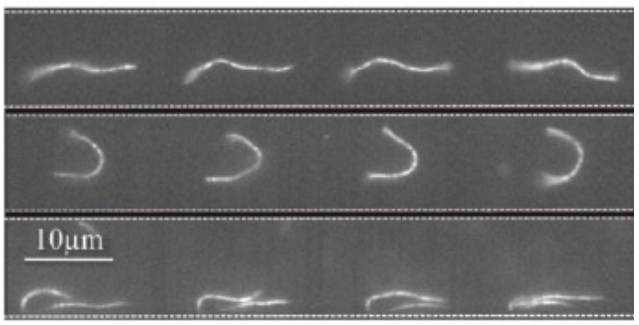

(b)

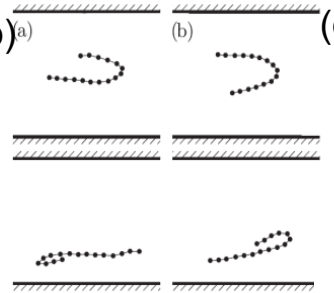

(e)

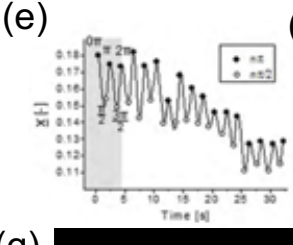

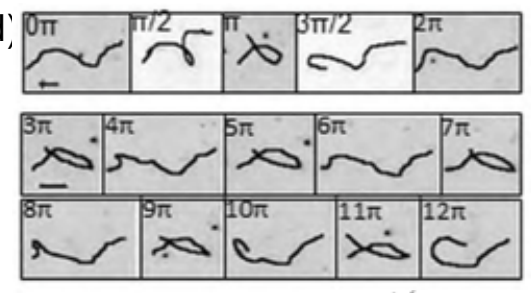

(f)
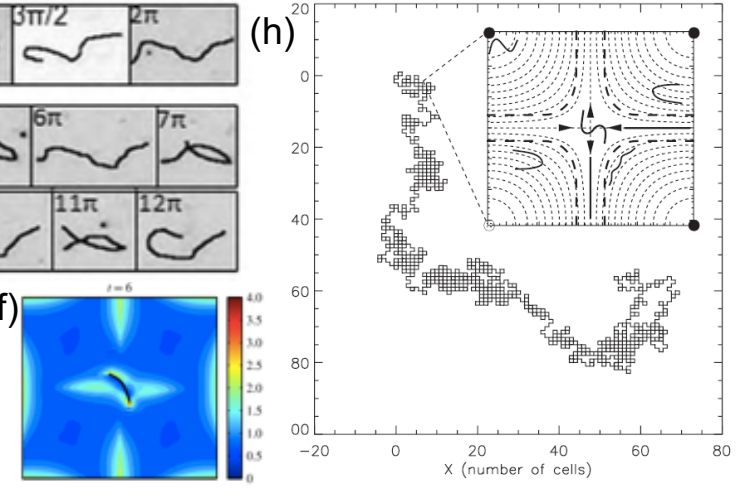

(g)

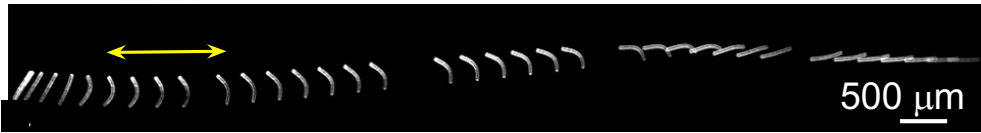

FIG. 5. Fibers in complex flows. (a), (b) \& (c) Fiber shapes in Poiseuille flow. (a) \& (b) From bead-spring models, (a; [16]; b; [115]) and (c) from experiments using actin filaments, [130]. (d) \& (e) Electrospun nanofilament in an oscillatory flow, [103]. (d), Different morphologies and (e) normalized filament-wall distance during successive oscillations. (f) Flexible fiber in cellular flow of a polymeric fluid, from simulations, [147]. The color scale indicates polymer stretching. (g) Trajectory of a flexible polymeric fiber confined by the top and bottom walls of a microchannel, [15]. (h) Simulations of the random walk of a flexible filament across a cellular flow, [150]. 
several groups have demonstrated, using bead numerical models ([16, 115]) or experiments with actin filaments ([130],Fig. 5c), that semi-flexible polymers show strong cross-stream migration in shear gradients and accumulate at preferred distances from channel walls. Similar predictions have been made for flexible fibers ([126]). Very recently [93] observed cross-stream migration using Brownian electrospun hydrogel nanofilaments in an oscillatory flow (Fig. 5d) and highlighted the dependance of migration dynamics on the initial fiber positioning.

\section{Confined flows}

Fiber transport in a Hele-Shaw cell (Fig. 2c) represents a very different situation. Fibers with a diameter comparable to the cell height are confined by the top and bottom walls and now evolve in 2D in a plug flow. Such confined microfabricated fibers (sec. II A) have been observed to bend into a C-shape facing backwards relative to the flow direction (Fig. 5e). At first sight it is surprising that a fiber transported at zero force in a plug-flow deforms, but this can be explained as follows: friction with the top and bottom walls decreases the fiber velocity compared to the average flow velocity and the fiber can be seen as a moving obstacle. The fiber thus creates a flow perturbation inducing an inhomogeneous force distribution along the fiber. [15] have evaluated the force distribution as a function of fiber confinement using modified Brinkman equations. The resulting fiber shapes are found to be in good agreement with experimental findings ([15]). The coupling between fiber deformation, drift, and interaction with the side walls ([92]) leads to complex trajectories and finally a preferred alignment with the flow direction (Fig. 5e).

\section{Cellular flows}

From their numerical study of fiber-flow interactions [150] predicted that flexible fibers could move as random walkers across a closed stream-line cellular flow (Fig. 5h). Fiber buckling, occuring close to the stagation points, yields due to its many degrees of freedom an effective randomness, allowing the fiber to cross streamlines and to be transported across the flow. The precise transport properties depend in a non-trivial way on fiber flexibility and length, as has also been shown in experiments ([139]). Numerical simulations by [81] addressed the role of Brownian fluctuations which were shown to increase trapping of filaments in vortices and thus decrease transport across the vortex array. [27] have numerically confirmed these findings. See [9] and [148] for a numerical study of two fibers interacting in such cellular flows and [147] for transport in a polymeric cellular flow (Fig. 5f.)

\section{SINGLE FIBERS MOVING UNDER EXTERNAL FORCES AND CONSTRAINTS}

\section{A. Sedimenting Fibers}

When flexible fibers are subject to external forces such as gravity, the coupling between the elastic and viscous forces can induce complex deformation and reorientation that strongly affects their macroscopic transport. Sedimentation is the classical example (though centrifugation would have similar consequences). The sedimentation of flexible fibers was first studied from the theoretical point of view by [146]. Numerical studies have since been conducted using bead models $([22,26,119,121])$ or slender-body theory $([73])$. Only very recently have experimental studies been performed $([84,114])$.

During sedimentation, fiber weight and viscous drag balance, and a rigid fiber settles at constant velocity and orientation. Hydrodynamic interactions along the fiber yield a viscous drag that is smaller in the middle than at the edges. For a flexible fiber, this inhomogenous drag deforms the fiber and leads to rotation. The theoretical work of [73] first explored how this affects settling trajectories. As the drag depends on fiber orientation, the rotation leads to a nontrivial coupling between horizontal and vertical translations (Fig. 6a). This occurs until the fiber has reached an equilibrium configuration characterized by a constant settling velocity and a bent shape, the middle being perpendicular to gravity independently of the release conditions (Fig. 6b). [119] have explored numerically the behavior of very flexible and very heavy filaments whose shape can become non-planar resulting in drift or helical trajectories (fig.6c).

The fiber shape at equilibrium depends on the relative magnitudes of the gravitational force and the elastic restoring force. The relevant control parameter is $B=F_{g} L^{2} / E$, with $F_{g}$ the gravitational force (Fig. 6d) ([22, 26, 84, 119]). For small $B$ the steady velocity is close to the horizontal settling velocity of a rigid fiber, while for large $B$, the fiber experiences large deformations and the velocity is close to the vertical settling velocity of a rigid fiber (Fig. 6e) ([26]). [84] recently confirmed this experimentally and identified the intermediate regime as one of continuous drag reduction 


\section{Trajectories}

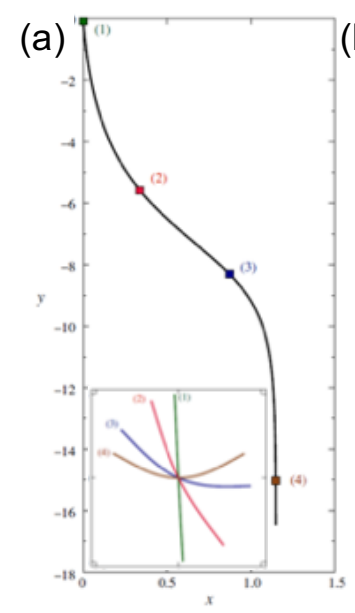

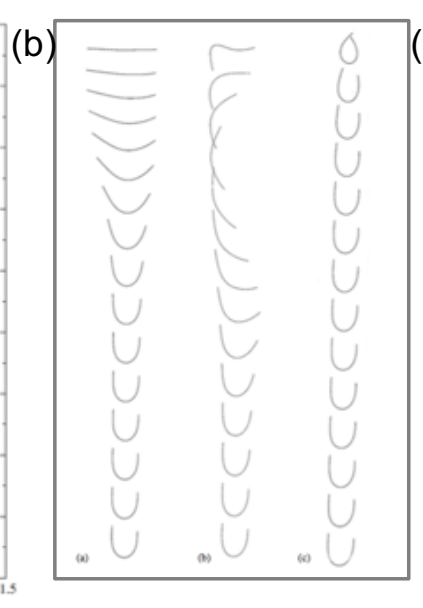

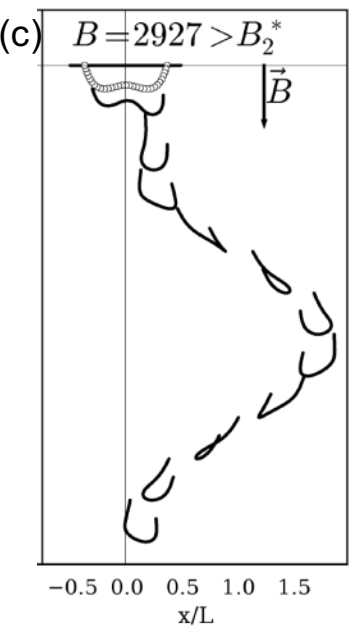

Equilibrium

Metastable shape

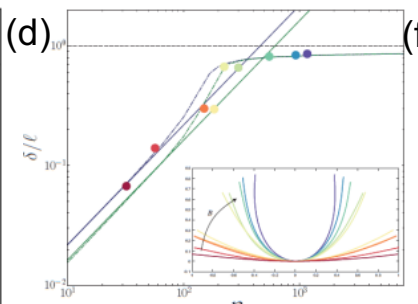

(f)
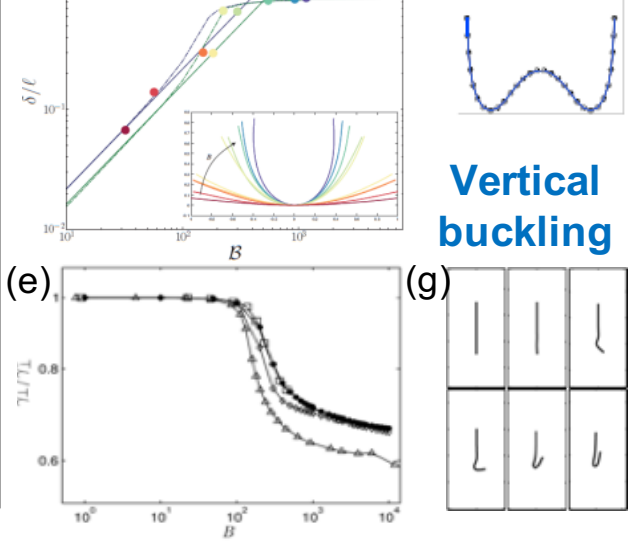

Vertical buckling

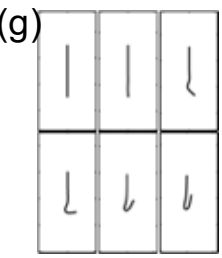

FIG. 6. Sedimentation of flexible fibers. (a) Trajectory of the fiber center of mass, insert, fiber shapes during sedimentation, [73]. (b) Chronophotographies of a fiber released with different initial conditions, [84]. (c) Complex trajectory of a fiber at very high $B$, [119]. (d) Maximal deformation of the fiber at steady state as a function of $B$, [84]. (e) Evolution of viscous drag at steady state as a function of $B$, [26]. (f) Example of metastable configuration from bead-spring model, [22]. (g) Buckling instability of the leading fiber half from simulations using SBT, [73].

through fiber reconfiguration. Defining an elasto-viscous number $\tilde{\eta}$ is not straightforward for this problem as the viscous forces are function of fiber shape.

The case of a fiber settling while aligned with gravity is very different: a inhomogenous fiber tension results from the inhomogenous viscous drag, and is compressive in the leading-half and extensile in the trailing part ([73]). Above a certain threshold of the control parameter, buckling may occur in the leading half (Fig. 6f).

\section{B. Anchored fibers}

Passive anchored fibers, such as the primary cilium ([149]), are found in biological systems, and can also form spontaneously under flow conditions as in the formation of biofilm streamers [30]. In engineered micro-fluidic flow geometries, fabricated anchored fibers can be used as flow sensors, or conversely the micro-fluidic flows can be used to measure the bending properties of unknown materials. Depending on the anchoring and flow orientation either buckling or bending can occur.

\section{Fiber buckling}

Using local SBT, [50] investigated the stability of elastic fibers when held against an impinging linear or quadratic stagnation point flow (Fig. 7a). One fiber end is either clamped or hinged at the wall (i.e. free to rotate with zero applied torque). To identify critical values in $\tilde{\eta}$ for buckling transitions they discretized the linearized dynamics equations and its boundary conditions and posed it as a generalized finite-dimensional eigenvalue problem for growth rates. For the clamped filament a first unstable bending mode was identified for both flow fields. The next unstable mode (in $\tilde{\eta}$ ) corresponds to buckling (Fig. 7a) with slightly different thresholds for the two flows. The hinged fiber is always unstable to rotation around its base, and its higher modes correspond to buckling, having significantly lower thresholds as compared to free fibers.

Experimental studies of fiber buckling in impinging flows are difficult as even a slight misalignment between the fiber base and the stagnation point leads to fiber bending (or rotation) instead of buckling. However, situations where long fibers remain either temporarily pinned, as observed for fibers flowing in rough fractures ([23]), or which experience a compressive flow only locally, as observed during continuous fiber fabrication in a widening microfluidic device ([98]), show buckling instabilities similar to those of anchored fibers. In particular, [98] show that such buckling can be used to form "crimped" microfibers with a well defined morphology (Fig. 7b). 


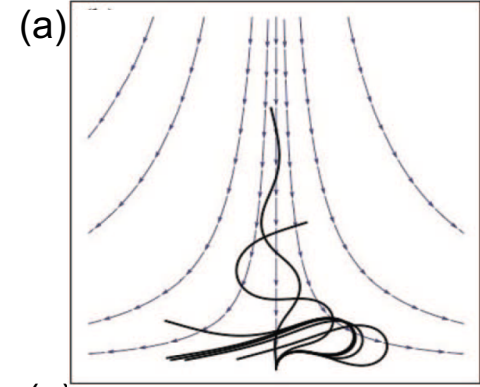

(e)

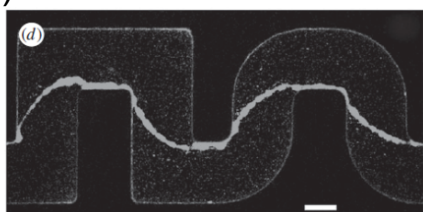

(b)

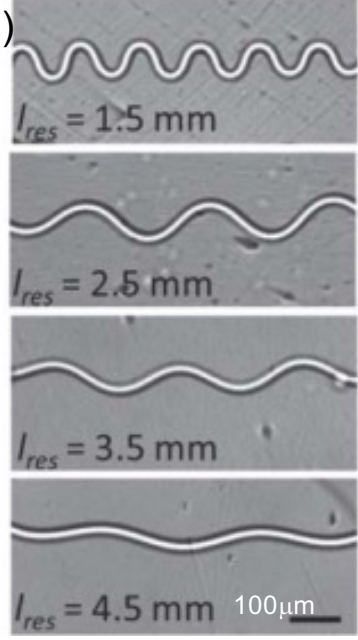

(c)

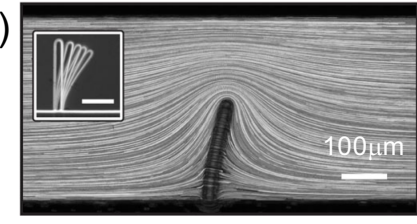

(f)

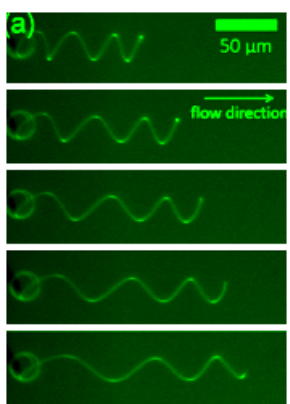

(g) (d)
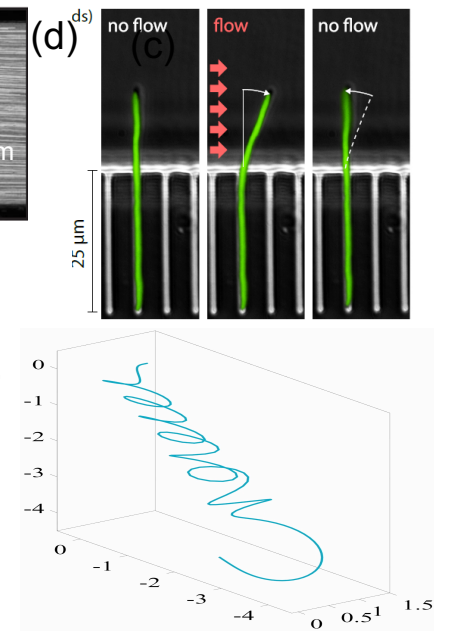

FIG. 7. Deformation of anchored fiber. (a) Time evolution of a clamped filament buckling in an impinging flow, [50]. (b) Buckling instability of a microfiber in a microchannel, [98]. (c) \& (d) Deformation of objects anchored perpendicular to the flow: a photopolymerized fiber confined by top and bottom walls(c; [140]), and single-cell Escherichia coli grown in a lateral slit,(d; [2]). (e) Biofilm streamers grown in a curved microchannel, [117]. (f) Micro-helix deformed by a parallel flow, [107]. (g) Clamped microtubule growing against a wall, from E. Nazockdast.

\section{Fiber bending}

The deformation of anchored fibers by viscous flows is, in a number of simple flow geometries, akin to a bending beam where viscous forces play the role of a gravitational load. Microscopic beam bending has been used by [32] to determine the bending modulus of an elastomeric fiber fabricated using the UV projection method introduced in Sect. II A. Here the fiber was confined between the top and bottom channel walls and held on both ends in a straight microchannel (Fig. 11). In this configuration flow takes place through the small gap above and below the fiber and the viscous force applied can be determined using a simple lubrication analysis.

The flow geometry is more complex when a confined fiber is attached to one wall and so partially blocks a confined channel. The resulting flow profile has been visualized (Fig. 7c) in experiment by [140] using fibers attached to the wall of a micro-channel and perpendicular to the flow direction. A theoretical model ([140]) gave insight into the competition between bending and leakage flow, showing good agreement with their experimental results, and was also used to measure fiber rigidity. By knowing its mechanical properties, such a fiber could be used as a flow sensor in micro-fluidic devices.

[2] studied the flow-induced bending of single-cell Escherichia coli growing from slits in the side-walls of a microfluidic channel (Fig. 7d). By applying a flow perpendicularly to the cells in their unconfined flow geometry, the experimental set-up corresponds again to a simple bending beam experiment where the force applied to the cell results from the viscous friction of the fluid. By estimating this viscous force and using linear elasticity theory, the authors were able to estimate the bending stiffness of the $E$. coli cells from the measured deflection. The simple hydrodynamic set-up gave values similar to those obtained using much more costly techniques such as AFM measurements.

Another biological example is the primary cilium. The primary cilium is a non-motile and flexible hair-like protrusion from a cell into the extracellular space and is found in a variety of vertebrate cells. The primary cilium is believed to act as a mechanoreceptor by bending in response to flow as is observed in kidney tubule cells. Its dynamics has been investigated in a combined experimental and theoretical study by [149].

More complex flow geometries are experienced by biofilm streamers that have been shown to grow into long, filamentous elastic structures $[116,117]$ under flow. The shape of elastic fibers within flows with curved streamlines has been determined numerically by [4] for fibers anchored, either hinged or clamped, at given positions near a twodimensional corner. This work shows that, due to tension and bending forces within the fibers, the fibers do not align with the flow but rather cross flow streamlines. This is in agreement with the experimental observations from $[116,117]$ shown on Fig. 7e. 


\section{Stretching of helical fibers}

The stretching by flow of very flexible anchored microhelices has been studied by [107]. Here the viscous drag represents a distributed loading leading to a nonuniform pitch distribution (Fig. 7f). The overall helix extension is well described by the FENE spring model, with the linear part simply given by the ratio of viscous to bending forces. The micro-scale helical ribbons with nano-scale thickness serve both as model systems for bacteria flagella and as very sensitive flow sensors.

\section{Actuated fibers}

Biological cilia and flagella are of central importance to biophysical processes like propulsion or embryogenesis $([6,10,58])$. Both are elongated fibers anchored to cellular membranes and which beat or rotate through the action of internal molecular motors (like the cilia covering the surface of micro-organisms; see [14]) or by rotating motors anchored at their base (like bacterial flagella; see [10]). Their interactions with the surrounding fluid medium lead to shape deformations to useful work like transport processes like propulsion or mucus clearance in the lung. The study of anchored filaments submitted to forces is also very important to understand the coupling between cytoskeletal filaments and molecular motors that occurs in the cell (e.g. $[124,138]$ ) during intracellular trafficking or to design micro-robots capable of motion in a fluid.

In the large literature on this topic, largely beyond the scope of this review, fibers are usually anchored to a wall but in some studies fibers are attached to an object small enough to be moved by the fiber motions (i.e. flagella and bacteria). This was studied at the micron-scale in two different experiments (Figs. 8a-b). Early to the field, [142] attached an actin filament to a bead that was periodically driven by an optical trap. The bead oscillations induced traveling waves on the fiber that generated propulsive force. In another realization of this phenomenon [31] built an artificial micro-swimmer from a chain of magnetic beads attached to a red-blood cell - only here to break symmetry - and actuated the chain with a time-dependent magnetic field.

In another class of studies, fiber motion is induced by the rotation of the anchoring point. [144] took inspiration from biology to model the deformations induced by rotation of a fiber around its axis in a viscous fluid. Rotation, twisting and bending combine to yield different regimes of rotation, one of them having the free end repelled from the axis. The interaction of two rotating fibers has been recently studied numerically and experimentally by [78] (Fig. 8d), who show that the induced rotational flows tend to bend the fibers around each other. If a fiber is tilted, at the anchoring point, relative to its rotation axis, the interaction with the fluid leads to the collapse of the fiber onto the rotation axis (Fig. 8c) while assuming a helicoidal shape that generates a propulsive force $([19,79,109])$. The rotation of a flexible helix can result in a propulsive force sufficiently large to induce buckling (Fig. 8e, [60]). The flexibility and buckling of the flagellar hook at the base of the flagellum plays an important role in the swimming of mono-flagellated bacteria where its buckling yields changes in swimming direction ([128]). Interactions between two weakly flexible helices result in bundling as has been shown experimentally by [68]. As a model for cilia, [51] studied the hydrodynamic coupling of immersed elastic fibers driven at their base by an applied torque. For two fibers their simulations and theory showed that bistability of in-phase and out-of-phase oscillations can arise from a coupling of elasticity and drive to flow.

Forced fibers can either be actuated locally or all along the body. The latter case has been experimentally and numerically modeled by magnetic colloid chains $([5,31,43])$. The former has been addressed in different experimental and numerical studies mainly with the forcing being applied to the anchoring point $([19,51,79,109,142,144])$, as already discussed, and typically yield oscillations. [25] recently studied tangential forcing at the fiber free-end as a model for the action of motor proteins on microtubules, and demonstrated a bifurcation to fiber beating via a buckling instability (Fig. 8f-g).

\section{MICRO-MACRO COUPLING AND COLLECTIVE BEHAVIOR}

We have thus far focused on single fibers (or a few) interacting with flows. However, in most industrial and natural settings flexible fibers are mostly found collectively and in suspension. Understanding the transport and rheology of fibrous suspensions at macroscopic length scales is of significant importance. In this chapter we review studies on the relationship between microscopic dynamics of suspended fibers and macroscopic rheology, as well as situations where fibers act collectively in their interactions with flow as in ciliary beds or in new flow control devices.

The main difficulty in studying fibrous assemblies or suspensions is the complex and long-ranged nature of hydrodynamic interactions (HIs). The importance of fiber interactions is defined by their average separation distance. In the dilute regime the fibers separation distance is much larger than their length: $n L^{3} \ll 1$ where $n$ is the fiber 

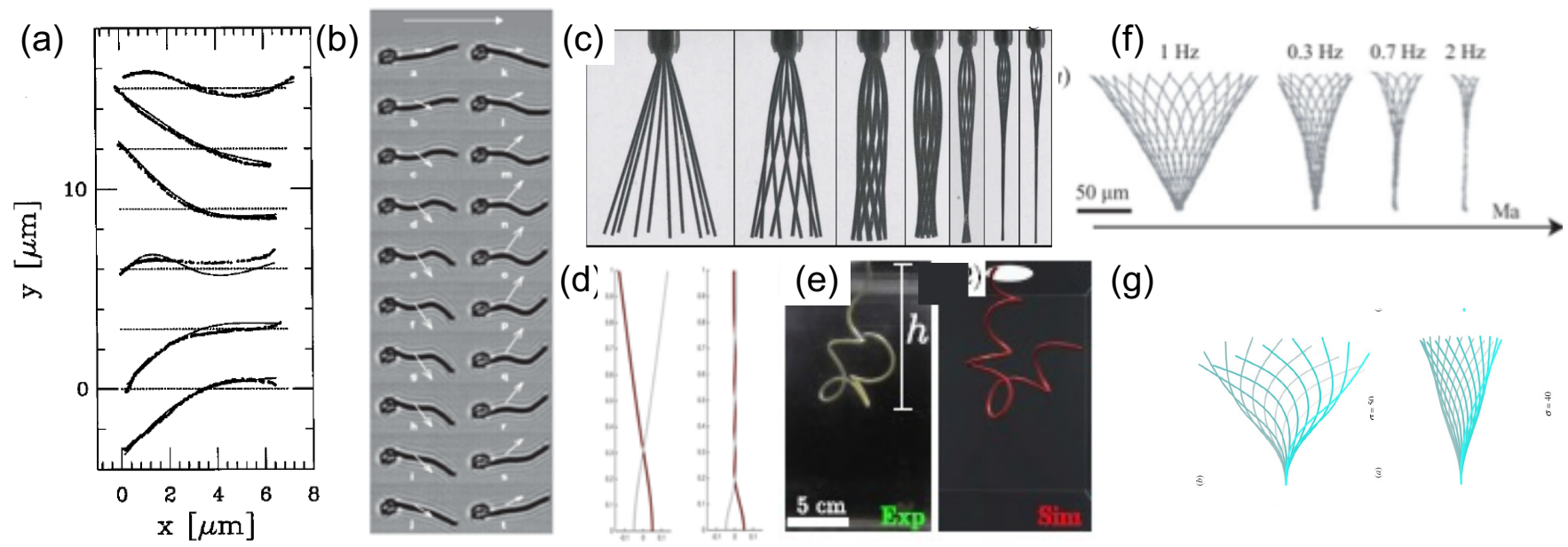

(g)

FIG. 8. Anchored and driven fibers. (a) \& (b) Fibers attached to a cargo. (a) A bead moved by an optical trap, [142]. (b) A magnetically actuated fiber propelling a red blood cell, [31]. (c) Rotation of a tilted elastic filament in a viscous bath at increasing rotation velocity, [19]. (d) Attractive interaction between two elastic fibers rotating around their own axes (left, experiments; right, numerical simulations, [78]. (e) Buckling of a rotating flexible helix, [60]. (f) \& (g) Shapes of beating fibers. (f) Magnetic fiber beating in a plane, [5]; (g) A simulation of a fiber actuated at its free-end by a "follower" force aligned with the fiber, [25].

number density. In this limit HIs can be neglected and for a suspension the ensemble behavior can be estimated from the dynamics of a single fiber interacting with the flow. Increasing the concentration causes HIs to be stronger. In the semi-dilute regime of $n L^{3}>\approx 1$, the distance between fibers becomes less than their length and both HIs and possibly steric interactions become important. With further increase of concentration the separation distance becomes comparable to the fiber radius and rotational motions are hindered. For suspensions this can result in formation of liquid crystalline phases ([29]), which is beyond the scope of this review.

\section{A. Rheology of fiber suspensions}

\section{Linear rheology for dilute non-Brownian suspensions}

The analysis of rheology for non-Brownian suspensions in oscillatory background shear flows is very similar to the buckling instability analysis in simple flows in §IV A 1. For small perturbations of fiber shape from a straight rod, whose dynamics are described by Eq. 11, Eq. (6) reduces to

$$
\tilde{\eta}\left(w_{t}-w \cos \tilde{\omega} t\right)=-w_{s s s}+2 \bar{T}_{s} w_{s}+\bar{T} w_{s s}, \quad \& \quad \bar{T}=-\frac{\tilde{\eta}}{4}\left(s^{2}-\frac{1}{4}\right) \cos \tilde{\omega} t
$$

where $w$ is the perturbation transverse to the fiber orientation in the plane of shear, and $\tilde{\omega}$ is the oscillation frequency normalized by the maximal shear rate $\dot{\gamma}$.

Perturbations will decay so long as $\tilde{\eta}<306.4$, the first bifurcation to buckling for the constant shear case. The long-time behavior will then be that of an oscillating rigid fiber which, because of time-reversibility, will yield only a viscous response under time average. For values of $\tilde{\eta}$ beyond the buckling transition fibers under compressive stress will buckle, with that buckling relieved in the reverse part of the cycle. Since elasticity is relaxational throughout the oscillation it remains unclear whether the long-time behavior supports persistent buckling. If not, the rheology will remain purely viscous.

\section{Linear rheology of dilute Brownian suspensions}

The linear viscoelastic behavior of semi-flexible polymers has been widely studied over the past two decades. Examples of these systems include worm-like polymers, cytoskeletal filaments and nanotubes. A detailed discussion of the literature in this limit is beyond the scope of this review, and readers are referred to [123]. Here we give a very brief summary relevant to this review. 
Rotational diffusion is the slowest time-scale for Brownian semi-flexible polymers, and is given by $\tau_{\text {rot }} \sim \mu L^{3} /\left(k_{b} T c\right)$. Another time-scale arises from curvature relaxation, given by $\tau_{\text {curv }}=8 \pi \mu L^{4} / E c$. The ratio of bending to rotational diffusion time-scales is $\tau_{\text {curv }} / \tau_{\text {rot }}=L / l_{p} \ll 1$. The fastest relaxation time is associated with tensile forces. In a rigid fiber the tension is set instantaneously. The presence of thermal forces induces shape fluctuations in semi-flexible polymers. Thus, the average end-to-end length of the polymer will be less than its full contour length and will change under external flows/forces. The tension time-scale is roughly defined as the time $\tau_{T}$ over which the end-to-end distance of the polymer reaches mechanical equilibrium under small external forces [123]. The tension dynamics is more involved, but has been studied in depth $([34,90,102])$. These studies find that $\tau_{\mathrm{T}} / \tau_{\text {curv }}=\left(L / l_{P}\right)^{4} \ll 1$. Thus, $\tau_{\mathrm{T}} \ll \tau_{\text {curv }} \ll \tau_{\text {rot }}$. This separation of time-scales, and the linear response in small deformation allows for analytical solutions of Eq. (9) in §III C, which is outlined in [123].

Having calculated the shape of the fiber, the time-dependent particle stress tensor is found by the force moment integral along the fiber: $\mathbf{S}=\int_{0}^{L} \mathbf{X}(s) \mathbf{f}(s) d s$, recalling that $\mathbf{f}=\mathbf{f}^{E}+\mathbf{f}^{B}$ where $\mathbf{f}^{E}=\left(T \mathbf{X}_{s}\right)_{s}-E \mathbf{X}_{s s s s}$, and $\mathbf{f}^{B}$ is given by Eq. (10). In dilute suspensions the interactions between the fibers are ignored, and the ensemble average stress reduces to $\boldsymbol{\Sigma}_{F}(t)=n\langle\mathbf{S}\rangle$. [123] used this expression to compute elastic and loss moduli in oscillatory shear flow for different values of $L / l_{p}$. Fig. 9a gives a schematic presentation of the tensile, bending, and thermal contributions to the time-dependent shear modulus relaxation in a step strain deformation (see Fig. 2 in [123]). At very short times where $t \ll \tau_{\text {curv }}$, the shear modules and its relaxation is determined by tensile forces, while for $t \sim \tau_{\text {curv }}$ all forces contribute equally to the rheology. Finally, at long times the behavior is dominated by thermal forces; thus the long-time behavior is viscous.

\section{Nonlinear rheology in shear flows}

Introducing flexibility to the fiber breaks time reversal symmetry of Stokes equation and changes the behavior qualitatively. [7] used local SBT to show that fiber buckling (see Fig. 4d) gave rise to positive first normal stress differences, and that the predicted threshold to buckling agreed well with the onset of positive first normal stress differences in shearing experiments of nylon fibers in glycerin [45].

[137] confirmed this observation in their nonlocal SBT simulations. Figure 9a shows the numerical predictions of Tornberg \& Shelley for first normal stress difference induced by a single flexible fiber in shear flow, $N_{1}=\mathbf{S}_{x x}-\mathbf{S}_{y y}$, during one rotation of the fiber (solid line). The results for a rigid rod are shown for comparison. To understand the physical basis for this behavior it helps to study the fiber's shape in three instances: (i) the beginning of deviation from rigid rod, (ii) when the deviations are maximized, and (iii) the return to a rigid rod behavior. These instances are labeled with open circles and are shown with their corresponding shapes in Fig. 9a. We see clearly that the deviation coincides with the buckling of fibers under compressional stress $(\mathbf{p} \cdot \mathbf{E} \cdot \mathbf{p}<0)$. As the fiber rotates to the extensional quadrant $(\mathbf{p} \cdot \mathbf{E} \cdot \mathbf{p}>0)$, it is stretched back by the straining component of the shear flow to its initial straight shape, and the corresponding stress approaches that of a rigid rod. Intuitively, buckling reduces the effective hydrodynamic length (the end-to-end distance) of the fiber, decreasing the stress magnitude in comparison with a rigid rod (recall that $|\mathbf{S}| \sim L^{3}$ ). Once the fiber moves to the extensional flow quadrant, it straightens and its effective length increase causes the stress magnitude to approach that of a rigid rod.

\section{Shear rheology of semi-dilute suspension}

Several aspects of the rheology of flexible fibers in semi-dilute regime remains poorly understood, due to the complexity of long-range HIs. To our knowledge, there is no large-scale simulation of flexible fibers in shear flow, with the exception of [137] who studied the suspension of $N_{F}=25$ flexible fibers in oscillatory shear flow. They observed that, at large enough shear rates, the fibers deform during the period they are in compressional quadrants of the flow. The fibers are then stretched back once they move to the extensional quadrants. However, they don't entirely relax, perhaps because of fiber-fiber interactions, and maintain strong buckling into the ensuing periods. They also observe that the total stored elastic energy is a strong function of the initial configuration of the filaments, which the authors relate to the small number of fibers in their simulations. For the same reason they did not report on the rheology of the suspension. To our knowledge, there is currently no prediction of shear viscosity and normal stresses vs shear rate.

On the experimental front, [59] studied the dynamics of individual actin filaments in semi-dilute solutions of mixtures of labelled and non-labelled filaments flowing in microchannels. They showed that steric hindrance reduces the tumbling rate significantly in semi-dilute solutions as compared to isolated filaments. Entanglements between filaments can also lead to anomalous or aborted tumbling events. The authors link the microscopic filament dynamics to the shear thinning viscosity of the semi-dilute solution via an effective diffusion coefficient. 
(a) Dilute suspension

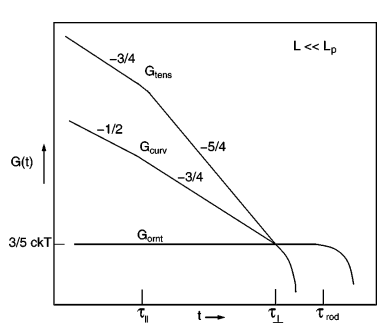

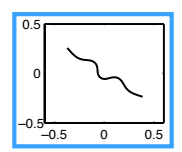
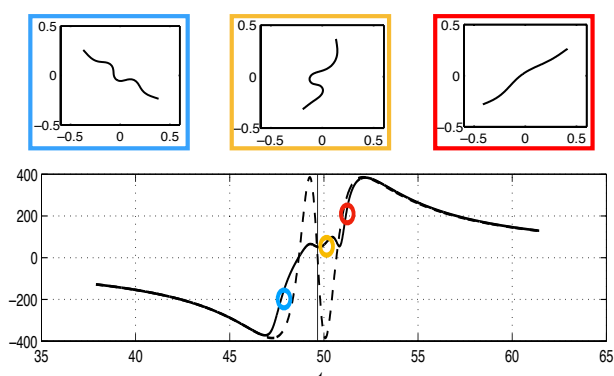

(c) Sedimentation

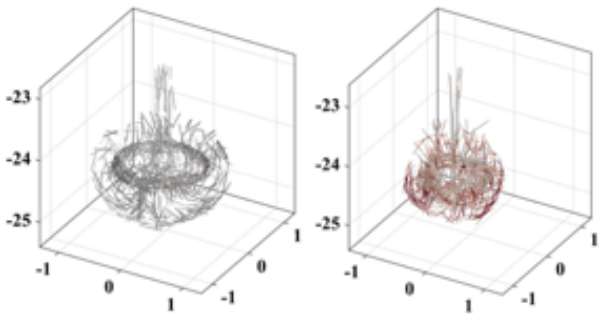

(c) Semi-dilute suspension

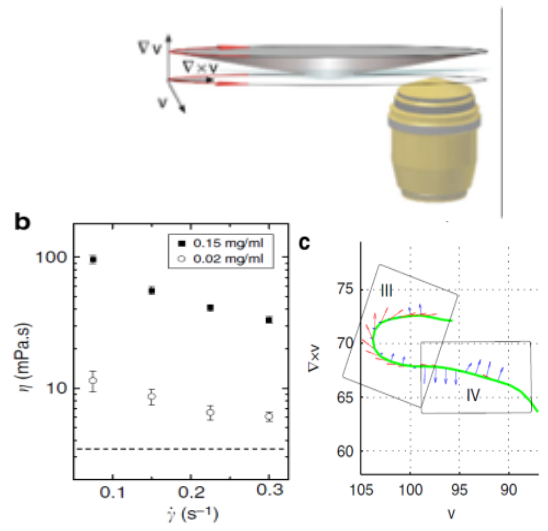

(d) Anchored

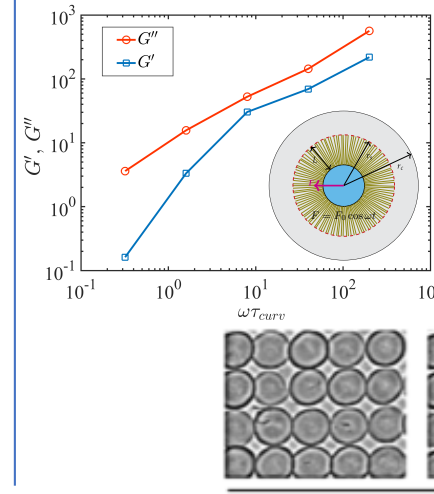

$0.2 \mathrm{~Hz}$
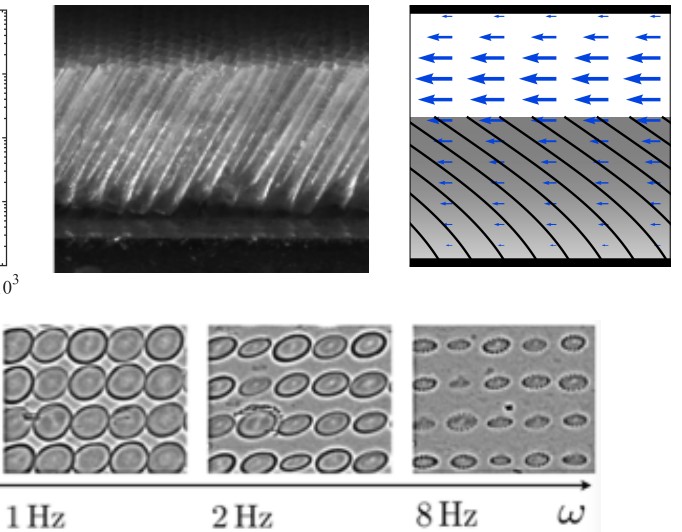

FIG. 9. Collective behavior. (a) Dilute suspensions: Left, asymptotic contributions of tensile, bending, and thermal forces to shear modulus for semi-flexible polymers ([123]). Right, first normal stress difference during shear for, solid curve, straight filaments, and dashed curve, buckling filament (shapes on the top row) ([137]). (b) Semi-dilute suspensions. Experimental set-up of [69] combining a rheometer and confocal imaging to measure the shear viscosity of semi-dilute actin suspension (left) and image in 3D the filament shape (right). (c) Sedimentation: Torus ring formed during the sedimentation of a cloud of 1024 flexible fibers (more flexible in the right graph than in the left graph), ([96]). (d) Anchored fibers: Top, left, rheology of a radially anchored flexible fiber shell, ([96]); Middle, array of tilted elastic fibers ([1]); Right, a simulation of flow through a anchored bed of flexible fibers using a continuum model of an elastic fiber Brinkman medium (from D. Stein \& M. Shelley, in preparation). Bottom, trajectory of the tips of many rotating magnetic fibers with increasing frequency, ([18]).

The effect of entanglements on the three-dimensional deformations of actin filaments in a semi-dilute solution was investigated experimentally by [69]. They used confocal microscopy on a cone-and-plate counter-rotating shear cell and were able to observe simultaneously microscopic filament dynamics and macroscopic flow properties (Fig. 9b). They showed that shear flow causes stretching and alignment of hair pin shaped filaments explaining the observed shear thinning.

A very different behavior is observed by [104] for concentrated suspensions of very long fibers. Using a microfluidic fabrication method (Fig. 1i) they produced concentrated suspensions of fibers with an aspect ratio greater than 100 and showed that gelation, resulting from the formation of mechanical interlocking of chains, is triggered by flow. This is also a very simple way to produce biocompatible hydrogels.

\section{B. Sedimentation of fiber assemblies.}

The sedimentation of particles in fluids has been a major area of research in suspension mechanics over the past several decades [49]. There have also been several experimental [56, 86] and numerical [12, 120, 136] studies on sedimentation of suspensions of rigid fibers. The complex behavior in sedimenting suspensions is due to HIs [13, 113]. For instance, sedimenting fiber suspensions form inhomogeneous clusters resulting in enhanced sedimentation rates of those clusters, in both numerical and experimental studies ([86, 120, 136]). [80] studied the effect of fiber flexibility under small deformations on sedimentation using a continuum theory, later followed by particle simulations [83]. These studies showed that flexibility can have both a stabilizing (no clusters) and destabilizing (cluster formation) 
effect, depending on the relative magnitudes of fiber flexibility and rotational diffusion.

Another interesting problem is the sedimentation of particle clouds. Experiments and simulations have used rigid rods and spheres to show that an initially spherical cloud evolves into a descending toroidal shape reminiscent of a high Reynolds number vortex ring. The torus eventually breaks into smaller clouds which evolve into tori ([77, 86, 87, 101]). [96] used nonlocal SBT to study clouds of $N_{F}=1024$ flexible fibers in a semi-dilute regime. Their observations were in general agreement with the previous experimental and computational studies on rigid rods (Fig. 9c). They observed no qualitative effect of fiber flexibility on the collective behavior of the cloud though flexibility does result in changes in the shape of the torus ring (manuscript in preparation). Fig. 9c shows the tori for two clouds with fibers of differing effective rigidities (65 in ratio). In short, increasing flexibility results in a smaller tori.

\section{Collective dynamics of anchored fibers.}

In many biological processes and industrial applications fiber assemblies are attached to substrates and particles. Detailed theoretical descriptions are still few, and a full accounting is beyond the scope of our review, but we discuss several interesting recent examples.

[1] have recently shown how to construct soft flow rectifiers by mounting dense beds of tilted flexible fibers onto the walls of a flow channel (Fig. 9d, right panel). When fluid is driven in the direction of the fiber tilt, the fiber bed depresses and facilitates flow. When fluid is pumped in the opposite direction the bed rises and occludes flow. The mechanical responses were quantified in a Taylor-Couette device, and rationalized theoretically using a homogenous Brinkman approximation for fluid flow through the bed.

In a related, but very different, work [96] studied the rheology of centrosomal arrays of flexible microtubules in eukaryotic cells. Using nonlocal SBT, they simulated the dynamics of flexible fibers radially anchored to a sphere moving under an external oscillatory force, $F=F_{0} \cos \omega t$ (schematic inset in Fig. 9d, top left). At low frequencies the response was purely viscous, and the fiber array was well-modeled as a homogeneous Brinkman medium. At higher frequencies, the force and velocity response moved out of phase, reflecting viscoelasticity, and they used the phase lag to approximate the elastic, $G^{\prime}$, and loss moduli, $G^{\prime \prime}$ (Fig. $9 \mathrm{~d}$ ). For a single fiber $G^{\prime \prime} / G^{\prime}$ has a minimum at: $\omega_{1}=\tau_{\text {curv }}^{-1}$. A minimum was also observed in the many-fiber simulation but at $\omega \approx 25 \tau_{\text {curv }}^{-1}$. In other words, HIs between the fibers decreased the relaxation time by a factor of $\approx 25$. An explanation is that the fibers create a poroelastic medium whose fluid penetration length decreases with increasing fiber number. Thus, bending modes with wavelengths above that of the penetration length are prohibited, resulting in shorter effective fiber length, and a much shorter relaxation time.

[95] used the same computational framework to study pronuclear positioning, an important transport process in eukaryotic cells. They showed that ignoring HIs leads to order-of-magnitude mispredictions of the necessary positioning forces. They also showed that different proposed models for the active forces gave rise to different cellular flows, and proposed flow measurement as a tool to differentiate between active mechanisms.

Beds of actively beating cilia are common in biology, propelling ciliated organisms, pumping fluid in the brain ([35]), performing selective filtering ([94]), and clearing mucus from the lungs. [88] carried out a 3D simulation of propagating metachronal waves in rows of pulmonary cilia. They considered a system where cilia sit primarily in a Newtonian viscous fluid, while the tip moves within a viscoelastic fluid modeling a mucus layer. They found that minimizing the work done by molecular motors results in synchronized beating of cilia, and suggested that HIs between cilia can lead to synchronized beating. [28] use a regularized Stokeslet method to study the dynamics of an infinite bed of driven cilia. They showed that metachronal waves not only enhance fluid transport above the bed, but increases mixing of fluid within it. Finally, in experiments [18] investigated the dynamics of extended arrays of artificial cilia driven by a precessing magnetic field. Whereas the dynamics of an isolated cilium was a rigid body rotation, collective driven beating results in a symmetry breaking of the precession patterns. The trajectories of the cilia are anisotropic and experience a significant structural evolution as the actuation frequency increases (Fig. 9d, bottom, left).

\section{SUMMARY AND OUTLOOK}

As should be clear, flexible fibers interacting with flowing liquids present a rich source of problems in fluid/structure interaction. They also present complicated phenomena, which require sophisticated experimental techniques to observe and measure, and complicated theories through which to understand them.

There are many areas of open inquiry, among them the interactions of fibers with complex media. Viscoelastic responses are typical of many biological environments, such as the reproductive tract [36] or inside of the cell [143]. There is a developing literature on swimming of microorganisms in viscoelastic fluids ([33, 134]). Using a 2D slenderbody actuated elastica model, [135] recently studied the role of body flexibility for undulatory swimming in viscoelastic 
fluids. There have been few if any studies of flexible fibers interacting with complex flows of complex fluids; Recent simulations of [147] (Fig. 5f) of fiber transport by cellular viscoelastic flow is the first of which we are aware. The fundamental theoretical difficulty is the necessity of evolving bulk elastic stresses via transport nonlinearities. This makes fluid-structure problems, much less those with multiple elastic bodies, very challenging for viscoelastic flows and morally equivalent to those for the Navier-Stokes equations. On the experimental side, it remains challenging to synthesize complex fluids with well-characterized (and simple!) rheological responses.

New kinds of mathematical coarse-grained descriptions need to be developed to describe collective behavior of flexible fibers in fluids, especially when hydrodynamic interactions are strong. One regime where progress is being made is when the fibers can be considered as well-aligned. In recent work, Stein \& Shelley (in preparation) have developed a continuum Brinkman-type model that captures the anisotropic drag from elongated flexible structures to compute the flow feedback to the bending and tensile response of a porous elastic medium $([89,133])$. Figure $9 \mathrm{~d}$ (top right) shows the model's result in simulating a soft flow rectifiern by the bending of a bed of tilted and anchored elastic fibers (Fig. 9d (top middle) from [1]).

\section{ACKNOWLEDGMENTS}

E.N. and M.J.S. acknowledge the support of the National Science Foundation, the National Institutes of Health, and the Department of Energy. A.L. acknowledges support from European Research Council Consolidator Grant 682367 under the European Union's Horizon 2020 program.

\section{LITERATURE CITED}

[1] Alvarado J, Comtet J, De Langre E, Hosoi AE. 2017. Nonlinear flow response of soft hair beds. Nature Physics 13:10141019

[2] Amir A, Babaeipour F, McIntosh DB, Nelson DR, Jun S. 2014. Bending forces plastically deform growing bacterial cell walls. Proceedings of the National Academy of Sciences 111:5778-5783

[3] Attia R, Pregibon DC, Doyle PS, Viovy JL, Bartolo D. 2009. Soft microflow sensors. Lab on a chip 9:1213-8

[4] Autrusson N, Guglielmini L, Lecuyer S, Rusconi R, Stone HA. 2011. The shape of an elastic filament in a two-dimensional corner flow. Physics of Fluids 23:063602

[5] Babataheri A, Roper ML, Fermigier M, du Roure O. 2011. Tethered fleximags as artificial cilia. Journal of Fluid Mechanics 678:5-13

[6] Babu D, Roy S. 2013. Left-right asymmetry: cilia stir up new surprises in the node. Open Biology 3:130052

[7] Becker L, Shelley M. 2001. The instability of elastic filaments in shear flow yields first normal stress differences. Phys. Rev. Lett. 87:198301

[8] Berthet H, du Roure O, Lindner A. 2016. Microfluidic Fabrication Solutions for Tailor-Designed Fiber Suspensions. Applied Sciences 6:385

[9] Bouzarth E, Layton A, Young YN. 2011. Modeling a semi-flexible filament in cellular Stokes flow using regularized Stokeslets. Int. J. Num. Meth. Biomed. Eng. 27:2021-2034

[10] Bray D. 2001. Cell Movements: From Molecules to Motility. Garland, N.Y.

[11] Broedersz CP, MacKintosh FC. 2014. Modeling semiflexible polymer networks. Reviews of Modern Physics 86:995

[12] Butler JE, Shaqfeh ES. 2002. Dynamic simulations of the inhomogeneous sedimentation of rigid fibres. Journal of Fluid Mechanics 468:205-237

[13] Butler JE, Snook B. 2018. Microstructural dynamics and rheology of suspensions of rigid fibers. Annual Review of Fluid Mechanics

[14] Camalet S, Jülicher F, Prost J. 1999. Self-Organized Beating and Swimming of Internally Driven Filaments. Phys. Rev. Lett. 82:1590-1593

[15] Cappello J, Duprat C, du Roure O, Nagel M, Bechert M, et al. 2018. Transport of flexible fibers in confined micro-channels. submitted :1-14

[16] Chelakkot R, Winkler RG, Gompper G. 2010. Migration of semiflexible polymers in microcapillary flow. EPL 91:14001

[17] Choi CH, Yi H, Hwang S, Weitz DA, Lee CS. 2011. Microfluidic fabrication of complex-shaped microfibers by liquid template-aided multiphase microflow. Lab Chip 11:1477-1483

[18] Coq N, Bricard A, Delapierre FD, Malaquin L, du Roure O, et al. 2011. Collective Beating of Artificial Microcilia. Phys. Rev. Lett. 107:014501

[19] Coq N, du Roure O, Marthelot J, Bartolo D, Fermigier M. 2008. Rotational dynamics of a soft filament: Wrapping transition and propulsive forces. Phys. Fluids 20:051703 
[20] Cortez R. 2001. The method of regularized stokeslets. SIAM J. Sci. Comp. 23:1204-1225

[21] Cortez R, Nicholas M, et al. 2012. Slender body theory for stokes flows with regularized forces. Communications in Applied Mathematics and Computational Science 7:33-62

[22] Cosentino Lagomarsino M, Pagonabarraga I, Lowe CP. 2005. Hydrodynamic induced deformation and orientation of a microscopic elastic filament. Physical Review Letters 94:1-4

[23] D'Angelo MV, Semin B, Picard G, Poitzsch ME, Hulin JP, Auradou H. 2009. Single Fiber Transport in a Fracture Slit: Influence of the Wall Roughness and of the Fiber Flexibility. Trans. Porous Med. 84:389-408

[24] Darnige T, Bohec P, Lindner A, Clément E. 2017. Lagrangian 3D tracking of fluorescent microscopic objects in motion Lagrangian 3D tracking of fluorescent microscopic objects in motion. Review of Scientific Instruments 055106

[25] De Canio G, Lauga E, Goldstein RE, Goldstein RE. 2017. Spontaneous oscillations of elastic filaments induced by molecular motors. Interface 14

[26] Delmotte B, Climent E, Plouraboué F. 2015. A general formulation of Bead Models applied to flexible fibers and active filaments at low Reynolds number. Journal of Computational Physics 286:14-37

[27] Deng M, Grinberg L, Caswell B, Karniadakis GE. 2015. Effects of thermal noise on the transitional dynamics of an inextensible elastic filament in stagnation flow. Soft Matter 11:4962-4972

[28] Ding Y, Nawroth J, McFall-Ngai M, Kanso E. 2014. Mixing and transport by ciliary carpets: A numerical study. Journal of Fluid Mechanics 743:124-140

[29] Doi M, Edwards S. 1978. Dynamics of rod-like macromolecules in concentrated solution. part 1. Journal of the Chemical Society, Faraday Transactions 2: Molecular and Chemical Physics 74:560-570

[30] Drescher K, Shen Y, Bassler BL, Stone Ha. 2013. Biofilm streamers cause catastrophic disruption of flow with consequences for environmental and medical systems. Proc. Nat. Acad. Sci. USA 110:4345-50

[31] Dreyfus R, Baudry J, Roper ML, Fermigier M, Stone HA, Bibette J. 2005. Microscopic artificial swimmers. NATURE 437:862-865

[32] Duprat C, Berthet H, Wexler JS, du Roure O, Lindner A. 2015. Microfluidic in situ mechanical testing of photopolymerized gels. Lab on a chip 15:244-52

[33] Elfring GJ, Lauga E. 2015. Theory of locomotion through complex fluids. In Complex fluids in biological systems. Springer, 283-317

[34] Everaers R, Jülicher F, Ajdari A, Maggs A. 1999. Dynamic fluctuations of semiflexible filaments. Physical review letters $82: 3717$

[35] Faubel R, Westendorf C, Bodenschatz E, Eichele G. 2016. Cilia-based flow network in the brain ventricles. Science 353:176-178

[36] Fauci LJ, Dillon R. 2006. Biofluidmechanics of reproduction. Annu. Rev. Fluid Mech. 38:371-394

[37] Fiore AM, Balboa Usabiaga F, Donev A, Swan JW. 2017. Rapid sampling of stochastic displacements in brownian dynamics simulations. The Journal of Chemical Physics 146:124116

[38] Fixman M. 1978. Simulation of polymer dynamics. i. general theory. The Journal of Chemical Physics 69:1527-1537

[39] Flores H, Lobaton E, Méndez-Diez S, Tlupova S, Cortez R. 2005. A study of bacterial flagellar bundling. Bull. Math. Bio. 67:137-168

[40] Forgacs OL, Mason SG. 1959. Particle motions in sheared suspensions IX. Spin and deformation of threadlike particles. J. Coll. Sci. 14:457-472

[41] Forgacs OL, Mason SG. 1959. Particle motions in sheared suspensions X. Orbits of flexible threadlike particles. J. Coll. Sci. 14:473-491

[42] Galambos PC, Forster F. 1998. An Optical Micro-Fluidic Viscometer, In Micro-Electro-Mechanicical System (MEMS), no. 66

[43] Gauger E, Stark H. 2006. Numerical study of a microscopic artificial swimmer. Physical Review E 74:1-10

[44] Gittes F, Mickey B, Nettleton J, Howard J. 1993. Flexural rigidity of microtubules and actin filaments measured from thermal fluctuations in shape. Journal of Cell Biology 120:923-934

[45] Goto S, Nagazono H, Kato H. 1986. The flow behavior of fiber suspensions in newtonian fluids and polymer solutions. Rheologica acta 25:119-129

[46] Götz T. 2000. Interactions of fibers and flow: Asymptotics, theory and numerics. Ph.D. thesis, University of Kaiserslautern, Germany

[47] Goubault C, Leal-Calderon F, Viovy JL, Bibette J. 2005. Self-Assembled Magnetic Nanowires Made Irreversible by Polymer Bridging. Langmuir 21:3725-3729

[48] Griffith B, Hornung R, McQueen D, Peskin C. 2007. An adaptive, formally second order accurate version of the immersed boundary method. J. Comp. Phys. 223:10-19

[49] Guazzelli É, Hinch J. 2011. Fluctuations and Instability in Sedimentation. Annual Review of Fluid Mechanics 43:97-116

[50] Guglielmini L, Kushwaha A, Shaqfeh ES, Stone HA. 2012. Buckling transitions of an elastic filament in a viscous stagnation point flow. Physics of Fluids 24:123601

[51] Guo H, Fauci L, Shelley M, Kanso E. 2018. Bistability in the synchronization of actuatedêmicrofilaments. Journal of Fluid Mechanics 836:304?323

[52] Hämäläinen J, Lindström S, Hämäläinen T, Niskanen H. 2011. Papermaking fibre-suspension flow simulations at multiple scales. J. Eng. Math. 71:55-79

[53] Harasim M, Wunderlich B, Peleg O, Kröger M, Bausch AR. 2013. Direct Observation of the Dynamics of Semiflexible Polymers in Shear Flow. Physical Review Letters 110:108302 
[54] Haward SJ, Oliveira MS, Alves MA, McKinley GH. 2012. Optimized cross-slot flow geometry for microfluidic extensional rheometry. Physical Review Letters 109:1-5

[55] Helgeson ME, Chapin SC, Doyle PS. 2011. Hydrogel microparticles from lithographic processes: Novel materials for fundamental and applied colloid science. Current Opinion in Colloid \& Interface Science 16:106-117

[56] Herzhaft B, Guazzelli É. 1999. Experimental study of the sedimentation of dilute and semi-dilute suspensions of fibres. Journal of Fluid Mechanics 384:133-158

[57] Hinch E. 1994. Brownian motion with stiff bonds and rigid constraints. Journal of Fluid Mechanics 271:219-234

[58] Hirokawa N, Tanaka Y, Okada Y, , Takeda S. 2006. Nodal flow and the generation of left-right asymmetry. Cell 125:33âï ¿ ï ¿?45

[59] Huber B, Harasim M, Wunderlich B, Kro M, Bausch AR. 2014. Microscopic Origin of the Non-Newtonian Viscosity of Semi fl exible Polymer Solutions in the Semidilute Regime. ACS Macro Letters 3:136

[60] Jawed MK, Khouri NK, Da F, Grinspun E, Reis PM. 2015. Propulsion and Instability of a Flexible Helical Rod Rotating in a Viscous Fluid. Physical Review Letters 115:1-5

[61] Jeffrey G. 1922. The motion of ellipsoidal particles immersed in a viscous fluid. Proc. Roy. Soc. A :102-161

[62] Jeong W, Kim J, Kim S, Lee S, Mensing G, Beebe DJ. 2004. Hydrodynamic microfabrication via "on the fly" photopolymerization of microscale fibers and tubes. Lab on a Chip 4:576-580

[63] Jiang H, Wu Y, Yang B, Zhao YP. 2014. Force-moment line element method for stokes flow around a slender body. Engineering Analysis with Boundary Elements 44:120-129

[64] Johnson R. 1980. An improved slender-body theory for Stokes flow. J. Fluid Mech. 99:411-431

[65] Jun Y, Kang E, Chae S, Lee SH. 2014. Microfluidic spinning of micro- and nano-scale fibers for tissue engineering. Lab on a Chip 14:2145-2160

[66] Kantsler V, Goldstein RE. 2012. Fluctuations, dynamics, and the stretch-coil transition of single actin filaments in extensional flows. Physical Review Letters 108:038103

[67] Keller J, Rubinow S. 1976. Slender-body theory for slow viscous flow. J. Fluid Mech. 75:705-714

[68] Kim MJ, Bird JC, Van Parys AJ, Breuer KS, Powers TR. 2003. A macroscopic scale model of bacterial flagellar bundling. Proceedings of the National Academy of Sciences of the United States of America 100:15481-15485

[69] Kirchenbuechler I, Guu D, Kurniawan Na, Koenderink GH, Lettinga MP. 2014. Direct visualization of flow-induced conformational transitions of single actin filaments in entangled solutions. Nature Communications 5:5060

[70] Kuei S, Słowicka AM, Ekiel-Jezewska ML, Wajnryb E, Stone HA. 2015. Dynamics and topology of a flexible chain: Knots in steady shear flow. New Journal of Physics 17

[71] Lauga E, Powers TR. 2009. The hydrodynamics of swimming microorganisms. Reports on Progress in Physics 72:096601

[72] Li D, Xia Y. 2004. Electrospinning of Nanofibers: Reinventing the Wheel? Advanced Materials 16:1151

[73] Li L, Manikantan H, Saintillan D. 2013. The sedimentation of flexible filaments. Journal of Fluid Mechanics 735:705-736

[74] Lim S, Peskin CS. 2004. Simulations of the whirling instability by the immersed boundary method. SIAM Journal on Scientific Computing 25:2066-2083

[75] Lindner A, Arratia PE. 2016. Preface to Special Topic: Invited Articles on Microfluidic Rheology. Biomicrofluidics 10:043301

[76] Liu Y, Chakrabarti B, Saintillan D, Lindner A, , du Roure O. 2018. Morphological transitions of elastic filaments in shear flow. Proceedings of the National Academy od Sciences 115 (38):9438-9443

[77] Machu G, Meile W, Nitsche LC, Schaflinger U. 2001. Coalescence, torus formation and breakup of sedimenting drops: experiments and computer simulations. Journal of Fluid Mechanics 447:299-336

[78] Man Y, Page W, Poole RJ, Lauga E. 2017. Bundling of elastic filaments induced by hydrodynamic interactions. Physical Review Fluids 2:123101

[79] Manghi M, Schlagberger X, Netz RR. 2006. Propulsion with a Rotating Elastic Nanorod. Phys. Rev. Lett. 96:68101

[80] Manikantan H, Li L, Spagnolie SE, Saintillan D. 2014. The instability of a sedimenting suspension of weakly flexible fibres. Journal of Fluid Mechanics 756:935-964

[81] Manikantan H, Saintillan D. 2013. Subdiffusive transport of fluctuating elastic filaments in cellular flows. Physics of Fluids 25:073603

[82] Manikantan H, Saintillan D. 2015. Buckling transition of a semiflexible filament in extensional flow. Physical Review E Statistical, Nonlinear, and Soft Matter Physics 92:041002

[83] Manikantan H, Saintillan D. 2016. Effect of flexibility on the growth of concentration fluctuations in a suspension of sedimenting fibers : Particle simulations. Physics of Fluids 28:013303

[84] Marchetti B, Raspa V, Lindner A, du Roure O, Bergougnoux L, et al. 2018. Deformation of a flexible fiber settling in a quiescent viscous fluid. Physical Review Fluids 3:104102

[85] Mercader C, Lucas A, Derre A, Zakri C, Moisan S, et al. 2010. Kinetics of fiber solidification. Proceedings of the National Academy of Sciences 107:18331-18335

[86] Metzger B, Guazzelli E, Butler JE. 2005. Large-scale streamers in the sedimentation of a dilute fiber suspension. Physical review letters 95:164506

[87] Metzger B, Nicolas M, Guazzelli É. 2007. Falling clouds of particles in viscous fluids. Journal of Fluid Mechanics 580:283301

[88] Mitran SM. 2007. Metachronal wave formation in a model of pulmonary cilia. Computers 83 structures 85:763-774

[89] Moeendarbary E, Valon L, Fritzsche M, Harris AR, Moulding DA, et al. 2013. The cytoplasm of living cells behaves as a poroelastic material. Nature materials 12:253 
[90] Morse DC. 1998. Viscoelasticity of concentrated isotropic solutions of semiflexible polymers. 2. linear response. Macromolecules 31:7044-7067

[91] Munk T, Hallatschek O, Wiggins CH, Frey E. 2006. Dynamics of semiflexible polymers in a flow field. Physical Review E 74:041911

[92] Nagel M, Brun PT, Berthet H, Lindner A, Gallaire F, Duprat C. 2018. Oscillations of confined fibres transported in microchannels. Journal of Fluid Mechanics 835:444?470

[93] Nakielski P, Pawłowska S, Pierini F, Liwińska W, Hejduk P, et al. 2015. Hydrogel nanofilaments via core-shell electrospinning. PLoS ONE 10:1-16

[94] Nawroth JC, Guo H, Koch E, Heath-Heckman EA, Hermanson JC, et al. 2017. Motile cilia create fluid-mechanical microhabitats for the active recruitment of the host microbiome. Proceedings of the National Academy of Sciences 114:9510-9516

[95] Nazockdast E, Rahimian A, Needleman D, Shelley M. 2017. Cytoplasmic flows as signatures for the mechanics of mitotic positioning. Molecular biology of the cell 28:3261-3270

[96] Nazockdast E, Rahimian A, Zorin D, Shelley MJ. 2017. A fast platform for simulating semi-flexible fiber suspensions applied to cell mechanics. Journal of Computational Physics 329:173-209

[97] Nguyen H, Fauci L. 2014. Hydrodynamics of diatom chains and flexible fibers. Submitted

[98] Nunes JK, Constantin H, Stone Ha. 2013. Microfluidic tailoring of the two-dimensional morphology of crimped microfibers. Soft Matter :4227-4235

[99] Nunes JK, Sadlej K, Tam JI, Stone Ha. 2012. Control of the length of microfibers. Lab on a Chip 12:2301

[100] Olson SD, Lim S, Cortez R. 2013. Modeling the dynamics of an elastic rod with intrinsic curvature and twist using a regularized stokes formulation. J. Comp. Phys 238:169-187

[101] Park J, Metzger B, Guazzelli É, Butler JE. 2010. A cloud of rigid fibres sedimenting in a viscous fluid. Journal of Fluid Mechanics 648:351-362

[102] Pasquali M, Shankar V, Morse DC. 2001. Viscoelasticity of dilute solutions of semiflexible polymers. Physical Review E 64:020802

[103] Pawøowska S. 2017. Lateral migration of electrospun hydrogel nanofilaments in an oscillatory flow. PloS one 12:e0187815

[104] Perazzo A, Nunes JK, Guido S, Stone HA. 2017. Flow-induced gelation of microfiber suspensions. Proceedings of the National Academy of Sciences of the USA

[105] Peskin C. 2002. The immersed boundary method. Acta Num. 11:479-517

[106] Pham JT, Lawrence J, Lee DY, Grason GM, Emrick T, Crosby AJ. 2013. Highly stretchable nanoparticle helices through geometric asymmetry and surface forces. Advanced Materials 25:6703-6708

[107] Pham JT, Morozov A, Crosby AJ, Lindner A, du Roure O. 2015. Deformation and shape of flexible, microscale helices in viscous flow. Physical Review E 011004(R):1-5

[108] Pozrikidis C. 1992. Boundary integral and singularity methods for linearized viscous flow. Cambridge University Press

[109] Qian B, Powers T, Breuer K. 2008. Shape Transition and Propulsive Force of an Elastic Rod Rotating in a Viscous Fluid. Phys. Rev. Lett. 100:078101

[110] Qu Z, Temel FZ, Henderikx R, Breuer KS. 2018. Changes in the flagellar bundling time account for variations in swimming behavior of flagellated bacteria in viscous media. Proceedings of the National Academy of Sciences

[111] Quennouz N. 2013. Ph.D. thesis, UPMC, Paris, France

[112] Quennouz N, Shelley MJ, du Roure O, Lindner A. 2015. Transport and buckling dynamics of an elastic fiber in a viscous cellular flow. Journal of Fluid Mechanics 769:387-402

[113] Ramaswamy S. 2001. Issues in the statistical mechanics of steady sedimentation. Advances in Physics 50:297-341

[114] Raspa V, Marchetti B, du Roure O, Lindner A, Bergougnoux L, et al. 2018. Dynamic of sedimentation of slender elastic filaments in a viscous liquid. Manuscript in preparation

[115] Reddig S, Stark H. 2011. Cross-streamline migration of a semiflexible polymer in a pressure driven flow Cross-streamline migration of a semiflexible polymer. The Journal of Chemical Physics 135:165101

[116] Rusconi R, Lecuyer S, Autrusson N, Guglielmini L, Stone Ha. 2011. Secondary flow as a mechanism for the formation of biofilm streamers. Biophys. J. 100:1392-9

[117] Rusconi R, Lecuyer S, Guglielmini L, Stone Ha. 2010. Laminar flow around corners triggers the formation of biofilm streamers. J. Roy. Soc. Interface 7:1293-9

[118] Saad Y, Schultz MH. 1986. Gmres: A generalized minimal residual algorithm for solving nonsymmetric linear systems. SIAM Journal on scientific and statistical computing 7:856-869

[119] Saggiorato G, Elgeti J, Winkler RG, Gompper G. 2015. Conformations, hydrodynamic interactions, and instabilities of sedimenting semiflexible filaments. Soft Matter

[120] Saintillan D, Darve E, Shaqfeh ES. 2005. A smooth particle-mesh ewald algorithm for stokes suspension simulations: The sedimentation of fibers. Physics of Fluids 17:033301

[121] Schlagberger X, Netz RR. 2005. Orientation of elastic rods in homogeneous Stokes flow. Europhysics Letters (EPL) 70:129-135

[122] Schroeder CM, Babcock HP, Shaqfeh ESG, Chu S. 2003. Observation of Polymer Conformation hysteresis in extensional flow. Science 301:1515-1519

[123] Shankar V, Pasquali M, Morse DC. 2002. Theory of linear viscoelasticity of semiflexible rods in dilute solution. Journal of Rheology 46:1111-1154

[124] Shelley MJ. 2016. The Dynamics of Microtubule / Motor-Protein Assemblies in Biology and Physics. Annual Review of Fluid Mechanics 48:487-506

[125] Silva PES, Vistulo de Abreu F, Godinho MH. 2017. Shaping helical electrospun filaments: a review. Soft Matter 13 
[126] Slowicka AM, Wajnryb E, Ekiel-Jezewska ML. 2013. Lateral migration of flexible fibers in Poiseuille flow between two parallel planar solid walls. Euro. Phys. J. E 36:9844

[127] Smith DJ. 2009. A boundary element regularized stokeslet method applied to cilia-and flagella-driven flow. Proceedings of the Royal Society A: Mathematical, Physical and Engineering Science 465:3605-3626

[128] Son K, Guasto JS, Stocker R. 2013. Bacteria can exploit a flagellar buckling instability to change direction. Nat. Phys. 9:494-498

[129] Stein DB, Guy RD, Thomases B. 2017. Immersed boundary smooth extension (ibse): A high-order method for solving incompressible flows in arbitrary smooth domains. Journal of Computational Physics 335:155-178

[130] Steinhauser D, Köster S, Pfohl T. 2012. Mobility Gradient Induces Cross-Streamline Migration of Semi fl exible Polymers. Macro Letters 1:541-545

[131] Stockie JM, Green SI. 1998. Simulating the Motion of Flexible Pulp Fibres Using the Immersed Boundary Method. J. Comp. Phys. 147:147-165

[132] Strelnikova N, Göllner M, Pfohl T. 2017. Direct Observation of Alternating Stretch-Coil and Coil-Stretch Transitions of Semiflexible Polymers in Microstructured Flow. Macromol. Chem. Phys. 218:1600474

[133] Strychalski W, Copos CA, Lewis OL, Guy RD. 2015. A poroelastic immersed boundary method with applications to cell biology. Journal of Computational Physics 282:77-97

[134] Sznitman J, Arratia PE. 2015. Locomotion through complex fluids: an experimental view. In Complex Fluids in Biological Systems. Springer, 245-281

[135] Thomases B, Guy RD. 2017. The role of body flexibility in stroke enhancements for finite-length undulatory swimmers in viscoelastic fluids. Journal of Fluid Mechanics 825:109-132

[136] Tornberg A, Gustavsson K. 2006. A numerical method for simulations of rigid fiber suspensions. J. Comp. Phys. 215:172196

[137] Tornberg AK, Shelley M. 2004. Simulating the dynamics and interactions of elastic filaments in Stokes flows. J. Comp. Phys. 196:8-40

[138] Trong PK, Doerflinger H, Dunkel J, St Johnston D, Goldstein RE. 2015. Cortical microtubule nucleation can organise the cytoskeleton of drosophila oocytes to define the anteroposterior axis. Elife 4

[139] Wandersman E, Quennouz N, Fermigier M, Lindner A, du Roure O. 2010. Buckled in translation. Soft Matter 6:5715-5719

[140] Wexler JS, Trinh PH, Berthet H, Quennouz N, du Roure O, et al. 2013. Bending of elastic fibres in viscous flows: the influence of confinement. J. Fluid Mech. 720:517-544

[141] Wiens JK, Stockie JM. 2015. Simulating flexible fiber suspensions using a scalable immersed boundary algorithm. Computer Methods in Applied Mechanics and Engineering 290:1-18

[142] Wiggins CH, Riveline D, Ott a, Goldstein RE. 1998. Trapping and wiggling: elastohydrodynamics of driven microfilaments. Biophys. J. 74:1043-60

[143] Wirtz D. 2009. Particle-tracking microrheology of living cells: principles and applications. Annual review of biophysics 38:301-326

[144] Wolgemuth CW, Powers TR, Goldstein RE. 2000. Twirling and Whirling: Viscous Dynamics of Rotating Elastic Filaments. Phys. Rev. Lett. 84:1623-1626

[145] Wu J, Aidun CK. 2010. A method for direct simulation of flexible fiber suspensions using lattice boltzmann equation with external boundary force. International Journal of Multiphase Flow 36:202-209

[146] Xu X, Nadim A. 1994. Deformation and orientation of an elastic slender body sedimenting in a viscous liquid. Physics of Fluids 6:2889-2893

[147] Yang Q, Fauci L. 2017. Dynamics of a macroscopic elastic fibre in a polymeric cellular flow. Journal of Fluid Mechanics 817:388-405

[148] Young YN. 2009. Hydrodynamic interactions between two semiflexible inextensible filaments in Stokes flow. Phys. Rev. E 79:046317

[149] Young YN, Downs M, Jacobs CR. 2012. Dynamics of the primary cilium in shear flow. Biophys. J. 103:629-39

[150] Young YN, Shelley M. 2007. A stretch-coil transition and transport of fibers in cellular flows. Phys. Rev. Lett. 99:058303

[151] Zhang Q, Zeng S, Lin B, Qin J. 2011. Controllable synthesis of anisotropic elongated particles using microvalve actuated microfluidic approach. Journal of Materials Chemistry 21:2466

[152] Zilz J, Schäfer C, Wagner C, Poole RJ, Alves Ma, Lindner A. 2014. Serpentine channels: micro-rheometers for fluid relaxation times. Lab on a chip 14:351-8

[153] Zografos K, Pimenta F, Alves MA, Oliveira MS. 2016. Microfluidic converging/diverging channels optimised for homogeneous extensional deformation. Biomicrofluidics 10 